\title{
1 Concerted cellular responses to type I interferon propel memory \\ 2 impairment associated with amyloid $\beta$ plaques
}

3 Ethan R. Roy ${ }^{1}$, Gabriel Chiu ${ }^{1}$, Sanming Li ${ }^{1}$, Nicholas E. Propson ${ }^{1,2}$, Hui Zheng ${ }^{1,3}$, Wei Cao ${ }^{1,3 *}$

5 1. Huffington Center on Aging, Baylor College of Medicine, Houston, TX, USA

6 2. Current address: Denali Therapeutics, South San Francisco, CA, USA

7 3. Department of Molecular and Human Genetics, Baylor College of Medicine, Houston, TX, USA

$15{ }^{*}$ Correspondence:

16 Wei Cao One Baylor Plaza, BCM 230, Baylor College of Medicine, Houston, TX 77030, USA

17 (713) 798-8523 wei.cao@bcm.edu 


3

4

5

6

7

\section{Abstract}

Despite well-documented maladaptive neuroinflammation in Alzheimer's disease (AD), the principal signal that drives memory and cognitive impairment remains elusive. Here, we reveal robust, age-dependent cellular reactions to type I interferon (IFN), an innate immune cytokine aberrantly elicited by $\beta$ amyloid plaques, and examine their role in cognition and neuropathology relevant to $A D$ in a murine amyloidosis model. Long-term blockade of IFN receptor rescued both memory and synaptic deficits, and also resulted in reduced microgliosis, inflammation, and neuritic pathology. Interestingly, microglia-specific IFN receptor ablation attenuated the loss of post-synaptic terminals, whereas IFN signaling in neural cells contributed to pre-synaptic alteration and plaque accumulation. Intriguingly, IFN pathway activation displayed a strong inverse correlation with cognitive performance, promoting selective synapse engulfment by microglia rather than amyloid plaques. Overall, IFN signaling represents a critical module within the neuroinflammatory network of $A D$ and prompts a concerted cellular state that is detrimental to memory and cognition.

\section{Keywords}

interferon; memory impairment, neuroinflammation; synapse; microglia; Alzheimer disease 


\section{Introduction}

Alzheimer's disease $(A D)$ is the main cause of dementia, characterized by memory

42 impairment. Hallmarked by the deposition of $\beta$-amyloid plaques and accumulation of

43 neurofibrillary tangles, AD pathogenesis manifests with complex interactions between different brain cell types (1). Collective histological, bioinformatic and molecular analyses have highlighted a perpetual activation of microglia, the brain resident immune cells, and remarkable connection of a number of $A D$ risk polymorphisms and rare variants to microglia and innate immunity $(2,3)$. Despite an overwhelming consensus on the importance of neuroinflammatory responses, the core signal that disrupts cognition and memory in AD is not yet well understood.

Recently, we described a prominent antiviral immune response by microglia in multiple murine amyloid $\beta$ models, as well as human $A D(4)$. At the center of this branch of innate immunity are type I interferons (IFNs) and a large number of IFN-stimulated genes (ISGs), which usually confer an antiviral state in host cells. However, more light is being shed on the functions of these molecules in sterile central nervous system (CNS) inflammation. Recently, Hur et al. reported that

54 IFITM3, an ISG, functions as an immune switch to increase $\gamma$-secretase activity, promoting APP cleavage and amyloid pathology (5). Meanwhile, microglial subsets with gene signatures of IFN response ("interferon-responsive microglia," or IRMs) have been identified from single-cell RNAseq (scRNA-seq) studies on murine amyloid $\beta$ models and human AD brains $(6,7)$. Moreover, polymorphisms in several ISGs were recognized as risk factor for AD (8), while upregulated IFN response was detected in $A D$ patients carrying the TREM2 $\mathrm{R} 47 \mathrm{H}$ variant (9). Given these significant findings, in-depth analysis of the functional contribution of IFN pathway to AD pathogenesis is warranted.

We previously focused on young 5XFAD mice, a widely-studied A $\beta$ model, in which microglia innately responded to amyloid fibrils harboring nucleic acids (NA), activated IFN 
65 we examined the accrual of IFN-activated microglia over time and assessed heterogeneity of IFN-

66 responsive microglia. To determine the effects of IFN on cognitive function, plaque pathology,

67 and neuroinflammation, we performed a long-term blockade in older 5XFAD mice with abundant

68 plaques. We further analyzed 5XFAD mice deficient of IFN receptor in different cell lineages to

69 reveal cell-type specific roles of IFN signaling. Overall, we find that IFN signaling via multiple cell

70 types is essential for memory impairment and synaptic damage during amyloidosis.

71

72 


\section{Results}

74

75

76

77

\section{Age-dependent cellular activation by IFN in amyloidosis}

Previously, we observed an age-dependent increase of NA-containing plaques in 5XFAD brains (4). Nucleic acids, when complexed to amyloid, serve as an immunogenic stimulus that elicits an IFN response (10). We thus detected microglia with active IFN signaling, marked by nuclear Stat1, exclusively near NA+ amyloid plaques in young 5XFAD brains. To comprehensively examine the brain cells activated by IFN, we first generated a reporter mouse line (MxG) by crossing Mx1-Cre mice with the ROSA26 ${ }^{\mathrm{mT} / \mathrm{mG}}$ strain, in which IFN exposure results in $M \times 1$ (a well-known ISG) promoter-driven permanent GFP expression in responsive cells (11). These mice were then bred into the 5XFAD background and examined at different ages to gauge IFN signaling in the brain.

At 3 months, only a small number of $\mathrm{GFP}^{+}$cells were detected, most of which were identified as plaque-associated microglia, making up about $21 \%$ of all microglia in plaque-bearing regions (Fig. 1a,b). By 5 months, GFP+ microglia became more prevalent and, by 11 months of age, $\mathrm{GFP}^{+}$cells represented a majority of the microglia in these plaque-rich regions. We previously showed that Axl protein, a receptor tyrosine kinase (RTK) and known ISG, is enriched in both Stat1 $1^{+}$microglia surrounding amyloid plaques in mice, and neuritic plaques in human brains (4). As expected, we observed significant overlap of GFP and expression of Axl in microglia (Fig. 1C). In $\beta$-amyloidosis, a subset of microglia adopt a disease-associated microglia (DAM) phenotype, marked by high Clec7a expression $(12,13)$. To estimate the heterogeneity of IFN responsiveness within the DAM population, we quantified proportions of microglia in plaqueloaded regions of 5-month-old 5XFAD;MxG brains using GFP and Clec7a expression, and found that roughly half of $\mathrm{Clec7a}^{+}$cells were $\mathrm{GFP}^{+}$(Fig. 1d). This is consistent with elevated ISG transcripts detected in bulk Clec7a $\mathrm{a}^{+}$microglia transcriptome from APP-PS1 mice (4), and more importantly reveals a distinct subpopulation within DAM marked by IFN responsiveness. 
Interestingly, small numbers of astrocytes and blood vessels also expressed GFP in 5- and 11month-old 5XFAD brains (Fig. S1a,b), suggesting IFN signaling goes beyond microglia amid accumulating CNS amyloidosis. Of note, we found that the MxG reporter yielded no GFP expression in neurons after direct brain administration of IFN, though numerous glial cells and blood vessels turned green (data not shown). Therefore, since neurons were unable to express the Mx1-Cre-dependent GFP readout, we later relied on other methods to detect IFN signaling in neurons.

These findings demonstrate a striking age-dependent accrual of IFN-activated brain cells, particularly microglia, and illuminate a degree of heterogeneity among plaque-associated microglia.

IFN blockade rescues memory and synaptic deficits without altering plaque load

To examine the role of IFN in memory impairment, we implanted osmotic pumps with ventricular cannulae to administer an antibody that specifically blocks signaling of type I IFN receptor (IFNAR) into 4-month-old 5XFAD mice for 30 days (Fig. 2a). Mice were then subjected to $\mathrm{Y}$ maze and novel object recognition (NOR) assays to evaluate cognitive aspects related to memory loss, before brain tissues were subjected to detailed histological examination and gene expression analysis. As shown in Figure 2b, 5XFAD mice receiving isotype control IgG failed to show spatial novelty preference in the $\mathrm{Y}$ maze, as well as discriminate between novel and familiar objects in the NOR assay, indicating severe deficits in both working memory and short-term

117 reference memory retrieval, respectively. In contrast, 5XFAD mice receiving blocking antibody 118 behaved comparably to non-transgenic control mice, suggesting a full restoration of the memory 119 deficits spurred by amyloid deposition.

We then performed histological characterization of the brain tissues to explore the 121 neurophysiological basis for the marked reversal of memory loss. Examination of synapse 
122 markers showed that 5XFAD mice administered control lgG had reduced levels of synaptophysin

123 and PSD95 proteins, which label pre- and post-synaptic terminals on excitatory neurons,

124 respectively, as well as in functional co-localization $(\leq 200 \mathrm{~nm})$ of these markers (Fig. 2c).

125 Consistent with the outcome of the cognitive assays, these proteins were both significantly

126 elevated in 5XFAD mice with IFN blockade.

To understand the impact of IFN blockade on amyloid pathology, we stained plaques with species, therefore modifying plaque burden. However, IFN blockade did not affect the amount of $A \beta$ taken up by microglia, nor decrease the level of microglial CD68, a lysosomal receptor involved in phagocytosis (Fig. S2b,c). synapses during amyloidosis, and suppression of the pathway is sufficient to restore these deficits despite abundant plaque pathology.

\section{IFN blockade reduces microgliosis, inflammation, and neuritic pathologies}

For a deeper understanding of how chronic IFN signaling affects cells in the plaque microenvironment, we examined glia and peri-plaque neuritic structures in detail. IFN blockade effectively reduced Stat1 in the nuclei of both plaque-associated microglia and neurons, indicating

141 broad suppression of excessive IFN signaling in these cells, thus validating the efficacy of the

142 blockade strategy (Fig. 3a). We saw a partial reduction of total lba1 ${ }^{+}$area with IFN blockade in 143 both cortex and subiculum, the latter an area with the earliest and densest plaque deposition (Fig.

144 3b). In contrast, astrocyte reactivity markers were not significantly affected in either region (Fig. 145 S3a,b). Further scrutiny of microglia showed that, on a per cell basis, IFN blockade significantly 
146

147

reduced the levels of $\mathrm{Axl}$ and Clec7a expression (Fig. 3c), altogether implying an attenuation of microglial activation.

We next analyzed gene expression in hippocampal tissues from the experimental cohorts and found that IFN blockade not only significantly lowered the expression of ISGs, such as Irf7, Ddx58 and Slfn8, as expected, but also decreased the levels of Clec7a, Tnf and Ccl4, suggesting a broader dampening effect on neuroinflammation in general (Fig. 3d).

Dystrophic neurites surrounding amyloid plaques represent another hallmark of AD. We found that both phospho-neurofilament ${ }^{+}\left(\mathrm{pNF}^{+}\right)$dystrophic axons and phosphorylated endogenous tau $\left(\mathrm{CP} 3^{+} \mathrm{pTau}\right)$ within dystrophic neurites were partially but significantly diminished by IFN blockade in 5XFAD brain (Fig. 3e,f), implying an IFN-mediated mechanism in promoting these pathologies.

\section{Selective microglial Ifnar1 ablation alters microglial activation and prevents post-synaptic loss}

All nucleated mammalian cells express the type I IFN receptor and thus can respond to the cytokine. Although our analysis identified microglia as the earliest and primary responder to IFN, how instrumental microglia are in mediating IFN's overall effects in the brain is not known. We bred Ifnar $1^{f / / f 1}$ with $\mathrm{C} \times 3 \mathrm{cr} 1-\mathrm{Cre} \mathrm{ERT2}^{\mathrm{ER}}$ mice, then crossed with the 5XFAD strain to generate mice lacking IFN receptor selectively in microglia (here termed 5XFAD;MKO). When FACS-sorted $\mathrm{CD}_{11} \mathrm{~b}^{+}$and Cd11b- cells from the brains of MKO mice were analyzed, significant reduction of Ifnar1 was detected, in conjunction with decreased ISGs, only in the Cd11 $\mathrm{b}^{+}$population, confirming the selective knockout in microglia (Fig. S4). Also consistent with this, Stat1 protein was noticeably absent in plaque-associated microglia in 5XFAD;MKO brains (Fig. 4a).

Similar to IFN blockade, microglia in 5-month-old 5XFAD;MKO mice showed a reduction of $\mathrm{lba1}{ }^{+}$area and significantly less AxI expression on a per cell basis (Fig. 4b). However, Clec7a 
and CD68 levels were not affected. 5XFAD;MKO mice expressed significantly less ISG transcripts, such as Irf7, Oas1 and Ifi2712a, while maintaining the expression of classical DAM markers, such as Clec7a, Trem2 and Cst7 (Fig. 4c). These findings suggest that microglial IFN signaling selectively regulates a subset of molecular changes observed in plaque-associated microglia.

Examination of synaptic markers revealed an unexpected effect - while both pre- and post-synapses were reduced in normal 5XFAD brains, PSD95 ${ }^{+}$puncta density, but not synaptophysin+, was restored in 5XFAD;MKO (Fig. 4d). We also examined dystrophic neuronal structures and found that pTau levels were significantly reduced by ablating microglia-specific IFN signaling, despite comparable axonal dystrophy (Fig. S5e). Altogether, these results hint at a selective function of IFN signaling in microglial activation and synapse modification.

\section{Neural Ifnar1 ablation reduces amyloid plaques and restores pre-synaptic terminals}

To understand the importance of IFN signaling in non-microglia cells in the brain, we bred Ifnar ${ }^{f / / f l}$ and Nestin-Cre mice to generate 5XFAD mice with the type I IFN receptor ablated in neuroectodermal-derived cells, including neurons and glial cells other than microglia (here termed 5XFAD;NKO). Consistent with the conditional knockout, Stat1 signal in 5XFAD;NKO neuronal nuclei was significantly reduced (Fig. 5a). Of note, we did not detect overt accumulation of LC3BII or protein hyper-ubiquitination in the brains of adult Ifnar $1^{\mathrm{fl} / \mathrm{l} l}$;Nestin-Cre mice as reported (14), nor decreased expression of endogenous or transgenic full-length amyloid precursor protein (APP) with conditional Ifnar1 ablation, as reported to be affected by germline Ifnar1 deletion (15) (Fig. S5a).

In contrast to IFN blockade and microglia-specific Ifnar1 ablation, 5XFAD;NKO mice at 5 months accumulated fewer ThioS $^{+}$and $6 \mathrm{E} 10^{+}$plaques in different brain regions (Fig. 5b). Measurement of $A \beta$ inside microglial $C D 68^{+}$vesicles indicated unaltered plaque phagocytosis by microglia (Fig. S5b). Since IFITM3 was shown to function as an inflammation-triggered switch to 
enhance $A \beta$ production (5), we investigated the possible involvement of this ISG. First, we confirmed a sensitive and dose-dependent induction of Ifitm3 protein in primary neurons by IFN $\beta$ (Fig. S5c). Further, we confirmed the upregulation of Ifitm3 protein in dystrophic neurites, a known site of heightened $A \beta$ production and release, as well as in astrocytes in 5XFAD brains (Fig. S5d). Interestingly, a selective diminution of Ifitm3 was detected in 5XFAD;NKO, but not 5XFAD;MKO, mice (Fig. 5c). Neural IFN signaling did not have a major impact on overall dystrophic neuronal structures (Fig. S5e). such that synaptophysin ${ }^{+}$puncta levels, but not PSD95 ${ }^{+}$, were restored, implying a neural-intrinsic and IFN-dependent regulation of pre-synaptic bouton density during disease (Fig. $5 \mathrm{~d}$ ). Activitydependent events shape neuronal networks in part by elimination of inactive synapses, a mechanism critical for proper configuration of circuits. In post-natal brain, Stat1 signaling at compared to 5XFAD (Fig. 5e). In accordance, fewer pStat $1^{+}$puncta were present in the nuclei of

212 CA1 neurons, which project to the subiculum (Fig. S5h). brain cells on plaque formation and synaptic pathology. 
manifestation, i.e. cognition and memory impairment, remains unclear. We thus constructed a database containing gene expression profiles, numerous neuropathological parameters, and behavioral outcomes from the cohort of 5XFAD mice that underwent IFN blockade treatment to 221 perform unbiased correlation analysis. As shown in Figure 6a, ordering profiled genes from 222 strongest negative to strongest positive correlates with performance in $Y$ maze revealed that ISGs 223 were heavily enriched among genes most strongly associated with poor cognition. IRF7 is a 224 master transcriptional regulator of type I IFN-dependent immune response (4). Irf7 levels were 225 negatively correlated with $\mathrm{Y}$ maze performance, highlighting a pathogenic effect of IFN pathway 226 on memory (Fig. 6a,e).

For neuropathology parameters, we found strong positive correlations of $\mathrm{Y}$ maze performance with PSD95/synaptophysin co-localized synapses, as well as with overall PSD95+ synapse density, and to a lesser extent with synaptophysin ${ }^{+}$synapse density (Fig. 6b).

Although amyloid plaques represent a cardinal pathology of $A D$, we did not observe significant correlation of $\mathrm{ThioS}^{+}$or $6 \mathrm{E} 10^{+}$plaque load with $\mathrm{Y}$ maze performance (Fig. 6c). Dystrophic axons, which were partially sensitive to IFN (Fig. 3e), displayed weak negative correlation with Y maze performance (Fig. 6d). Reactive astrocytes are known to participate in neurodegenerative processes. However, readouts of GFAP protein signal and C3 mRNA, which

235 is primarily produced by reactive astrocytes, did not correlate with memory capacity in this cohort 236 (Fig. 6c,d).

Although microglial reactivity is highly influenced by $\beta$ amyloidosis, whether activated states of these cells protect or harm the brain function remains controversial. We found that both overall microgliosis marked by Iba1 levels and DAM generation marked by Clec7a levels within 240 microglia showed strong inverse correlations with Y maze performance (Fig 6e). 
Hence, these findings suggest a potent negative impact of IFN and microglia on memory and cognition under the context of amyloid deposition.

243 IFN signaling promotes microglia-mediated synaptic engulfment differential cellular requirements for IFN signaling (Figs. 4, 5) in mid-stage 5XFAD mice, we sought to further investigate microglia in synapse modification.

First, synaptic engulfment assays showed that microglia in 5-month-old 5XFAD brain selectively engulfed enhanced amounts of PSD95+ puncta, an activity dependent on extracellular

253 IFN (Fig. 7a) and necessarily mediated by IFN receptor in microglia (Fig. 7b). Consistent with this, more PSD95 was detected inside GFP+ microglia over GFP- counterparts in plaque-rich regions of $5 X F A D ; M x G$ mice at 5 months (Fig. 7c). In contrast, no enhanced synaptophysin+ ${ }^{+}$signal was detected inside microglia from 5XFAD brains, with or without IFN signaling, compared to control

257 mice (Fig. 7a, b), suggesting a selective post-synaptic elimination by microglia, persisting at different stages of disease.

We previously showed that IFN-activated microglia rapidly remove dendritic spines in a complement C3-dependent manner (4). Although IFN was sufficient in inducing many members

261 of the complement cascade in wild-type mice, blockade of extracellular IFN or genetic IFN 262 receptor ablation in 5XFAD mice did not reduce complement transcription (Fig. S7a,b), consistent 263 with unchanged C3 protein in astrocytes (Fig. S3b). This indicates that signals other than IFN may 264 play a role in chronic complement activation in older 5XFAD mice. 
Perineuronal nets (PNN) are extracellular matrix structures that enwrap and stabilize neuronal synapses, loss of which in 5XFAD was shown to be mediated by microglia (17). Although Wisteria floribunda agglutinin (WFA) staining confirmed a significant reduction of PNN structures in disease, IFN blockade did not appear to affect their levels (Fig. S7c), excluding a direct link between IFN and PNN modification.

Axl is a member of the TAM (Tyro3, Axl, and Mer) family RTKs that play important roles

271 in phagocytosis of apoptotic cells (18). Recently, a plaque-centric expression pattern of TAM

272 receptors and their ligand Gas6 was reported to engage microglia with amyloid plaques in a 273 largely Mer-dependent manner (19). Given the high sensitivity of microglial AxI to IFN signaling, 274 we investigated its relation to synapses, together with Mer and Gas6. Employing high275 magnification confocal imaging, we detected specific, punctate signals for Gas6 as well as both 276 Axl and Mer in wild-type brain (Fig. S7d), which interestingly displayed non-random co-localization 277 with synaptic puncta (Fig. S7e), indicating a physiological interaction of TAM molecules with 278 synapses. In diseased brain, we observed notable Gas6 deposition on amyloid plaques and 279 enhanced Mer expression in plaque-associated microglia (Fig. S7f,g), in agreement with Huang 280 et al (19). However, unlike Axl, Mer expression in microglia, as well as extent of Gas6 deposition 281 on plaques, were not IFN-dependent. At the synaptic structures, we found substantial Gas6 282 deposition on PSD95 $^{+}$synaptic terminals in 5XFAD brains, which was accompanied by 283 significantly increased Axl, but not Mer, co-localization with PSD95 (Fig. 7d). To visualize the 284 physical relationship between Axl and synapses, we analyzed dendritic spines of 5XFAD mice 285 containing the Thy1-eGFP reporter. High-magnification confocal imaging revealed the formation 286 of contact points between $\mathrm{GFP}^{+}$dendritic spines and $\mathrm{AxI}^{+}$microglial processes, which were 287 significantly more frequent along dendrites in 5XFAD mice (Fig. 7e), substantiating a direct contact of Axl with synapses. 
To explore the role of Axl receptor in synapse uptake, we examined PSD95 engulfment

290 by different subpopulations of microglia from control and 5XFAD brains, particularly Clec7a

291 plaque-associated microglia with varying expression of Axl, and detected robust per-cell

292 correlation of Axl and PSD95 occupancy in microglia (Fig. 7f). To test whether Axl and/or Gas6

293 localization to synapses is dependent on IFN signaling, we measured the frequency of Axl/PSD95

294 and Gas6/PSD95 co-localization in 5XFAD mice treated with IgG or alFNAR, and found that 295 colocalization of both AxI receptor and Gas6 ligand to synapses was reduced with IFN blockade, 296 a finding which was mirrored by microglial conditional Ifnar1 deletion (Fig. 7g), suggesting a 297 reversible, IFN-induced post-synaptic engulfment machinery in microglia during disease. In line 298 with this, Axl protein and mRNA levels displayed a strong positive correlation with PSD95 299 engulfment, and negative correlations with PSD95 density and Y maze performance (Fig. 7h, $300 \mathrm{S6a}$ ). Altogether, these data pinpoint an IFN-instructed synapse elimination program in microglia 301 that compromises memory. 


\section{Discussion}

Beyond antiviral function, type I IFN is linked to cognitive and neuropsychiatric dysfunction in various clinical contexts $(4,20)$. Previous studies describe that IFN modifies the brain through microglia activation, neural stem cell dysfunction, and disruption of whole-brain functional network connectivity $(4,21)$. Of the neuropathological features of $A D$, synapse loss appears early and correlates most strongly with dementia, and thus represents a key step of the disease process (22). Our current study reveals for the first time discrete and coordinated actions of IFN-stimulated brain cells in compromising synapses, the central cause of memory impairment, under the sterile inflammatory condition initiated by AD pathology (Fig S8).

A growing number of microglia populations are being identified by scRNA-seq analyses, revealing different activation states under various physiological or pathological conditions (23). While cells enriched with DAM markers were identified first, IRMs were subsequently recognized as a distinct subset of microglia arising in $\mathrm{AD}$ and brain aging $(6,7,24)$. Moreover, a microglial proteome analysis revealed that IFN pathway was activated early and persisted in murine $A \beta$

317 models (25), in line with our findings that microglial IFN response universally accompanies brain

318 amyloidosis in vivo (4). Using a genetically-encoded IFN-responsive reporter system, we documented an age-dependent, brain-wide, and profound accrual of brain cells responding to IFN signaling activation in the 5XFAD model. In young mice, a sparse population of microglia were the principal IFN-responsive cell-type, consistent with the results obtained with Stat1 staining (4). By 5 months, despite overwhelming presence of $\mathrm{NA}^{+}$plaques, no more than half of plaqueassociated $\mathrm{Clec} \mathrm{a}^{+}$microglia expressed GFP, revealing an interesting aspect of microglial heterogeneity. Given the higher percentage of cells turning green at older age, microglia seem to be activated by IFN continuously as amyloidosis progresses. It is also worth noting that over $90 \%$ 326 of $\mathrm{GFP}^{+}$microglia retained Clec7a expression, which implies that microglia maintain DAM 327 markers after IFN activation. As $\mathrm{GFP}^{+}$microglia accumulated alongside the increasing plaque 
load, other brain cells also became GFP+, revealing a more complex IFN response than previously appreciated. Many brain cell types participate in plaque formation and neuritic pathology, a process marked by a multicellular co-expression network of plaque-induced genes (PIGs) (26). We found that 22 of the 57 PIG module are CNS ISGs $(4,27,28)$, many of which overlap with the markers of DAM and neurotoxic reactive astrocytes (29) (Fig. S9), highlighting a profound influence of IFN in the dysregulated cellular network in the vicinity of plaques.

We obtained apparently conflicting results on whether IFN signaling affects plaque pathology: blocking IFN receptor did not (Fig. 2d), while neural Ifnar1 deletion partially reduced plaque load (Fig. 5b). The latter observation was correlated with significantly tempered Ifitm3 expression, in keeping with the activity of Ifitm3 in promoting APP cleavage and amyloid pathology (5). Paradoxically, Ifitm3 was unaltered with IFN blockade (Fig. S2d), implying a difference between extracellular IFN blocking and genetic ablation. One possible explanation comes from clinical observation with therapeutic aIFNAR antibodies that IFN has more persistent effects in cells devoid of negative IFN regulators ISG15 and USP18 (30). Interestingly, cortical neurons do not express Isg15 or Usp18 (both are ISGs) even after IFN exposure, a contrast to microglia (27, 28). Given the sensitive neuronal response of Ifitm3 induction by IFN (Fig. S5c), it is plausible that, under IFNAR blockade, residual IFN receptor signaling was sufficient to maintain the levels of Ifitm3. Of note, not all neuronal IFN signaling escaped extracellular blockade, as neuronal pStat1 and total Stat1 proteins were similarly reduced by antibody-mediated blockade (Figs. S2e, 3a) and genetic ablation of Ifnar1 in neural cells (Fig. 5a,e).

Another unique pathological hallmark of $A D$ is the swollen pre-synaptic dystrophic neurites surrounding amyloid plaques, which accumulate APP as well as $\beta$ - and $\gamma$-secretases, and serve as localized sites of $A \beta$ generation and release (31, 32). As we have shown (Fig. 5), neural IFN signaling was required for Ifitm3 expression, which is known to enhance $\gamma$-secretase activity. Remarkably, $\beta$-secretase expression is also reportedly regulated by interferon and Stat1 $(33,34)$, 
implying a sweeping effect of IFN on APP processing. Overall, our results support a feed-forward $A \beta$-plaque-IFN-A $\beta$ loop whereby inflammation stimulates factors that exacerbate $A D$ pathology.

We discovered that synaptophysin ${ }^{+}$boutons were selectively diminished by IFN signaling in neural-derived cells (Fig. 5d), in sharp contrast to the regulation of post-synaptic densities (Fig. 4d). A Jak2-Stat1 axis was recently identified as a major neuron-autonomous determinant to eliminate inactive synapses in vivo (16). Interestingly, Stat1 functions not only as a negative regulator of spatial memory formation in wild-type mice, but is also a key mediator of $A \beta$-induced learning and memory deficits $(35,36)$. We found increased pre-synaptic pStat1 in 5XFAD, which was reduced upon IFN blockade or ablation (Figs. 5e, S2e). Collectively, the experimental evidence strongly supports a novel neuronal IFN-Stat 1 axis that pathogenically modulates the pre-synapse in AD (Fig S8).

While microglia prune synapses during normal CNS development, excessive removal can result in pathological synapse loss in diverse neurological and neuropsychiatric diseases (37). In $\beta$-amyloidosis models, germline C3 deficiency protects from loss of synapses and neurons (38, 39), and microglia engulf C1q-tagged post-synaptic components early in the disease (40). Despite the strong relationship between IFN and complement in young 5XFAD mice, we found unexpectedly that IFN signaling became dispensable in eliciting complement expression in 5month-old animals, likely reflecting the influences of other prevailing proinflammatory signals. It should be noted that, in the same cohort, IFN blockade was effective to blunt Tnf and Clec7a expression, similar to the treatment effects in 10- to 12-month-old APP ${ }^{\text {NL-G-F }}$ mice (4).

Besides complement, microglia use myriad other surface receptors to engulf or otherwise limit synapses (41-44). Interestingly, several synapse-eliminating receptors recognize a common neuronal cue (45): phosphatidylserine (PS), a well-known "eat-me" signal for phagocytosis. The principal myeloid phagocytic receptors, Axl and Mer, detect PS exposed on apoptotic cells via 


\section{7}

378

their ligand Gas6, which displays high affinity towards PS (18). In CNS, Mer facilitates astrocytic phagocytosis of synapses in developing and adult brain (46), and can also engage plaques in $A D$ (19). Yet, the function of Axl in brain, despite its prominent upregulation in plaque-associated microglia in $A D$, is unknown. Intriguingly, we discovered a highly IFN-dependent AxI expression in microglia surrounding amyloid plaques (Fig 3,4), As reported (19), we found Mertk mRNA positively correlated with dense-core plaques (Fig. S6c). Contrary to Mer, IFN blockade and microglial Ifnar1 deletion effectively reduced Axl levels but failed to modify the plaques, in line with $A x I$ deficiency in APP/PS1 model (19). On the other hand, we demonstrate for the first time direct contact between Axl and synapses, which were highly tagged by Gas6 in $\beta$-amyloidosis, and enrichment of synaptic material inside $\mathrm{Axl}^{+}$microglia, all of which were IFN-dependent. While Mer expression displayed no association with either memory or synapse levels, Axl abundance was robustly and inversely correlated with memory performance (Fig. S6a,b). Interestingly, both soluble AXL and GAS6 levels increase in cerebrospinal fluid of AD patients $(47,48)$, consistent with the elevated Axl expression in human AD (4)(Fig. S7h). These intriguing findings warrant further characterization of a pathogenic involvement of microglial AxI in AD.

We present evidence that IFN signaling plays a role in phosphorylation of endogenous tau at neuritic plaques (Fig. 3), which constitutes a major type of AD-relevant tau pathology and, notably, has been shown to enable AD-tau spreading in vivo (49). Although uncertain how IFN modulates tau together with other peri-plaque dystrophies, we were intrigued by the report that increased IRMs were associated with heightened endogenous tau phosphorylation in Trem2deficient APP/tau double transgenic mice (50). Hence, the mechanism by which IFN-mediated signaling connects amyloid and tau pathologies in AD, and whether IFN pathway represents a feasible therapeutic target, are of great interest. 
402 The study was funded by NIH grants AG057587 (W.C. and H.Z.); AG020670 (H.Z.), AG062257

403 (H.Z.), NS093652 (H.Z.), BrightFocus ADR A20183775 (W.C.) and Brown Foundation 2020

404 Healthy Aging Initiative (W.C.). We acknowledge technical assistance from Haiying Liu, Nadia 405 Aithmitti, and Bianca Contreras, and express gratitude to Dr. Andre Catic for generously providing 406 MxG founder mice.

407

\section{Author Contributions}

409 Conceptualization, E.R.R. and W.C.; Methodology, E.R.R., G.C. and S. L.; Formal Analysis, 410 E.R.R..; Investigation, E.R.R., G.C. and S. L.; Resources, N.E.P.; Writing- Original Draft, E.R.R., 411 and W.C.; Writing- Review \& Editing, E.R.R. and W.C.; Visualization, E.R.R.; Supervision, W.C.;

412 Project Administration, W.C.; Funding Acquisition, H.Z., and W.C.

414 Declaration of Interests

415 The authors declare no competing interests. 
Fig. 1: Progressive IFN signaling in 5XFAD brain.

a, GFP expression upon Mx1-Cre-driven recombination in the $\mathrm{mT} / \mathrm{mG}$ reporter line crossed to the 5XFAD model. Representative images of 5XFAD and nTg control brains at 3 months $(n=3$ control, $n=3$ 5XFAD), 5 months ( $n=3$ control, $n=3$ 5XFAD), and 11 months ( $n=2$ control, $n=2$ 5XFAD)

421 of age, showing age-dependent expansion of IFN-responsive GFP+ cells throughout plaque422 bearing regions (scale bar, $250 \mu \mathrm{m}$ ).

423 b, Representative confocal images of tissues from a co-labelled with lba1 with quantification 424 below, showing an IFN-responsive $\mathrm{GFP}^{+}$subset of plaque-associated microglia that expand in an 425 age-dependent manner ( 3 months: $n=75$ cells from 3 animals; 5 months: $n=301$ cells 3 animals; 42611 months: $n=140$ cells from 2 animals; scale bar, $20 \mu \mathrm{m})$.

427 c, Representative image of a 5-month-old 5XFAD animal ( $n=3$ animals) revealing that Axl is 428 expressed primarily in the $\mathrm{GFP}^{+}$subset of microglia (solid arrowheads) over the GFP- subset 429 (hollow arrowheads). Scale bar, $20 \mu \mathrm{m}$.

d, Representative images from 5 month old 5XFAD brains showing both GFP $^{+}$and GFP- $^{-}$cells 431 (left) and varying $\mathrm{Axl}$ expression (middle) among the plaque-associated $\mathrm{Clec}^{+} \mathrm{a}^{+}$subset of 432 microglia, revealing significant heterogeneity of MGnD microglia in the plaque environment (scale 433 bars, $15 \mu \mathrm{m})$. (Right) Quantification of microglial subtypes at 5 months using Iba1, Clec7a, and 434 IFN-responsive GFP reporter expression ( $n=408$ cells from 3 animals). 
a, Schematic depicting long-term i.c.v. administration of alFNAR or IgG control to 4-month-old control or 5XFAD mice via mini osmotic pumps with brain ventricular cannulae. After 30 days mice were subjected to behavioral assays to assess memory loss and then harvested for brain tissue analyses. NOR: novel object recognition.

b, Results of behavioral assays. Ctrl + IgG, $n=13$ animals; Ctrl + alFNAR, $n=11$ animals; 5XFAD + IgG, $n=11$ animals; 5XFAD + alFNAR, $n=11$ animals. Data represent means and s.e.m. Statistics for $Y$ maze were performed with ordinary one-way ANOVA $\left(P<0.001, \mathrm{~F}_{42}=33.46\right)$ and Bonferroni's multiple-comparisons test. ns, not significant; ${ }^{* \star *} P<0.001$. Statistics for NOR were performed with two-way ANOVA $\left(P<0.001, F_{84}=28.36\right)$ and Tukey's multiple-comparisons test. ns, not significant; ${ }^{* * *} P<0.001$.

c, Representative high-magnification images of pre-synapses, marked by synaptophysin, and post-synapses, marked by PSD95, in subicula of treated Ctrl and 5XFAD animals (scale bar, 3 $\mu \mathrm{m})$. Quantification of relative densities of synaptic markers, and the density of functional synapse pairs (<200nm distance between puncta). Ctrl + IgG, $n=13$ animals; Ctrl + alFNAR, $n=11$ animals; $5 X F A D+\operatorname{lgG}, n=11$ animals; 5 XFAD + aIFNAR, $n=11$ animals. Data represent means

457 and s.e.m. Statistics were performed with ordinary one-way ANOVA (Syp: $P<0.001, F_{42}=6.659$; 458 PSD95: $P<0.001, F_{42}=15.36$; Co-localized pairs: $P<0.001, F_{42}=15.98$ ) and Bonferroni's 459 multiple-comparisons test. ns, not significant; ${ }^{* *} P<0.01 ;{ }^{* * *} P<0.001$. administration of $\alpha$ IFNAR using $6 \mathrm{E} 10$ antibody to mark $A \beta$ fibrils, and thioflavin $S$ to mark dense core plaques (scale bar, $500 \mu \mathrm{m}$ ). Quantifications of plaque load for both markers in relevant brain 
bioRxiv preprint doi: https://doi.org/10.1101/2021.11.01.466525. this version posted November 3, 2021. The copyright holder for this preprint (which was not certified by peer review) is the author/funder, who has granted bioRxiv a license to display the preprint in perpetuity. It is made available under aCC-BY-NC-ND 4.0 International license.

463 regions. 5XFAD + IgG, $n=11$ animals; $5 X F A D+$ alFNAR, $n=11$ animals. Data represent means

464 and s.e.m. Statistics were performed with two-tailed $t$-tests. ns, not significant.

465

466

467

468

469

470

471

472

473

474

475

476

477

478

479

480

481

482 

neuritic tau.

a, Representative images of Stat1 levels, a marker of IFN activation, in the brains of 5XFAD animals treated with $\operatorname{lgG}$ ( $n=11$ animals) or alFNAR $(n=11$ animals). Insets show isolated Stat1 channels from boxed areas, highlighting microglia (solid boxes) and $\mathrm{NeuN}^{+}$neuronal nuclei (dashed boxes). Scale bar, $30 \mu \mathrm{m}$.

b, Images of Iba1 staining in the cortex and hippocampus of treated 5XFAD animals (scale bar, $500 \mu \mathrm{m})$, and quantifications of $\%$ lba1 area. Ctrl + IgG, $n=13$ animals; Ctrl + alFNAR, $n=11$ animals; 5XFAD + IgG, $n=12$ animals; 5 XFAD + aIFNAR, $n=12$ animals. Data represent means and s.e.m. Statistics were performed with ordinary one-way ANOVA (CTX: $P<0.001, F_{44}=50.84$; SUB: $\left.P<0.001, \mathrm{~F}_{44}=74.94\right)$ and Bonferroni's multiple-comparisons test. ns, not significant; ${ }^{\star} P<0.05 ;{ }^{* *} P<0.01 ;{ }^{* * *} P<0.001$.

c, Representative images of plaque-associated microglia in treated 5XFAD animals expressing $\mathrm{Axl}$ and Clec7a (isolated in insets; scale bar, $30 \mu \mathrm{m}$ ), with quantification of microglial occupancy of both markers. Ctrl + IgG, $n=13$ animals; Ctrl + alFNAR, $n=11$ animals; 5 XFAD + IgG, $n=11$ animals; 5XFAD + alFNAR, $n=11$ animals. Data represent means and s.e.m. Statistics were performed with ordinary one-way ANOVA (Axl: $P<0.001, \mathrm{~F}_{42}=42.24$; Clec7a: $P<0.001, \mathrm{~F}_{42}=$ 41.77) and Bonferroni's multiple-comparisons test. ns, not significant; ${ }^{* *} P<0.01$; ${ }^{* *} P<0.001$.

d, Gene expression alterations with alFNAR treatment as measured by Nanostring analysis. Ctrl $502+\operatorname{lgG}, n=9$ animals; Ctrl + alFNAR, $n=8$ animals; 5XFAD + IgG, $n=8$ animals; 5XFAD +

503 alFNAR, $n=10$ animals. Data represent means and s.e.m. Statistics were performed with 504 ordinary one-way ANOVA (Irf7: $P=0.007, \mathrm{~F}_{31}=4.923 ; D d x 58: P<0.001, \mathrm{~F}_{31}=9.283 ;$ Slfn8: $P$ $505<0.001, \mathrm{~F}_{31}=9.086 ;$ Clec7a: $P<0.001, \mathrm{~F}_{31}=42.99 ;$ Tnf: $P=0.036, \mathrm{~F}_{31}=3.228 ;$ Ccl4: $P<0.001$, 
$\left.\mathrm{F}_{31}=18.83\right)$ and Bonferroni's multiple-comparisons test. ns, not significant; ${ }^{\star} P<0.05 ;{ }^{* *} P<0.01$;

507 ${ }^{\star * *} P<0.001$ neurofilament ${ }^{+}\left(\mathrm{pNF}^{+}\right)$dystrophic axons (DAs) surrounding methoxy-X04+ amyloid plaques in subicula of treated animals (scale bar, $20 \mu \mathrm{m}$ ). 5XFAD + IgG, $n=11$ animals; 5XFAD + alFNAR, $n=12$ animals. Data represent means and s.e.m. Statistics were performed with two-tailed $t$-tests. ${ }^{\star *} P<0.01$.

f, Representative image of $\mathrm{CP}_{13}{ }^{+}$aggregated tau foci inside LAMP1+ DNs in the subiculum of a 514 5XFAD animal (insets show isolated channels for CP13 and total tau; scale bar, $30 \mu \mathrm{m}$ ), and 515 quantification of $\mathrm{CP} 13^{+}$occupancy in DNs after treatment with IgG or alFNAR. 5XFAD + IgG, $n=$ 51611 animals; 5 XFAD $+\alpha$ IFNAR, $n=12$ animals. Data represent means and s.e.m. Statistics were 517 performed with two-tailed $t$-test. ${ }^{* *} P<0.01$. 
Fig. 4: Selective microglial IFNAR ablation alters reactive microglial phenotype and prevents post-synaptic loss.

a, Representative images of Stat1 expression in the plaque environment of 5XFAD animals with microglia-specific deletion of Ifnar1 (“5XFAD;MKO”; $n=8$ animals) compared to Ifnar1-sufficient 5XFAD animals ("5XFAD"; $n=7$ animals) (insets show isolated Stat 1 channel in plaqueassociated microglia; scale bar, $30 \mu \mathrm{m})$.

b, Quantifications of total \% lba1+ area and activation marker occupancy (CD68, Axl, and Clec7a) on a per microglia basis by region. For Iba1, Axl, and Clec7a: Ctrl, $n=9$ animals; Ctrl;MKO, $n=$ 6 animals; 5XFAD, $n=7$ animals; 5XFAD;MKO, $n=8$ animals. For Cd68: Ctrl, $n=4$ animals; Ctrl;MKO, $n=4$ animals; 5XFAD, $n=4$ animals; 5XFAD;MKO, $n=4$ animals. Data represent means and s.e.m. Statistics were performed with ordinary one-way ANOVA (Iba1, CTX: $P<0.001$, $\mathrm{F}_{34}=73.93$; Iba1, SUB: $P<0.001, \mathrm{~F}_{34}=212.6$; Cd68, CTX: $P<0.001, \mathrm{~F}_{12}=38.45$; Cd68, SUB: $P$ $<0.001, \mathrm{~F}_{12}=72.61 ; \mathrm{Axl}, \mathrm{CTX}: P<0.001, \mathrm{~F}_{26}=12.52 ; \mathrm{Axl}$, SUB: $P<0.001, \mathrm{~F}_{26}=43.68 ; \mathrm{Clec7a}$, CTX: $P<0.001, F_{26}=8.893$; Clec7a, SUB: $\left.P<0.001, F_{26}=10.29\right)$ and Bonferroni's multiplecomparisons test. ns, not significant; ${ }^{* \star *} P<0.001$. $n=4$ animals; 5XFAD, $n=12$ animals; 5XFAD;MKO, $n=4$ animals. Data represent means and s.e.m. Statistics were performed with ordinary one-way ANOVA (Irf7: $P<0.001, \mathrm{~F}_{29}=9.300$; Oas 1: $P<0.001, \mathrm{~F}_{29}=13.41 ;$ Ifi27/2a: $P<0.001, \mathrm{~F}_{29}=11.79 ;$ Clec7a: $P<0.001, \mathrm{~F}_{29}=27.77$; Trem2: $P$ $<0.001, \mathrm{~F}_{29}=18.19 ;$ Cst7: $\left.P<0.001, \mathrm{~F}_{29}=30.97\right)$ and Bonferroni's multiple-comparisons test. ns, not significant; ${ }^{\star} P<0.05 ;{ }^{* *} P<0.01 ;{ }^{* *} P<0.001$. and PSD95, respectively, in subicula of Ctrl ( $n=9$ animals), Ctrl;MKO ( $n=6$ animals), 5XFAD ( $n$

$550=7$ animals), and 5XFAD;MKO ( $n=8$ animals). Scale bar, $3 \mu \mathrm{m}$. Quantification of relative synaptic 
bioRxiv preprint doi: https://doi.org/10.1101/2021.11.01.466525. this version posted November 3, 2021. The copyright holder for this preprint (which was not certified by peer review) is the author/funder, who has granted bioRxiv a license to display the preprint in perpetuity. It is made available under aCC-BY-NC-ND 4.0 International license.

551 puncta densities. Data represent means and s.e.m. Statistics were performed with ordinary one-

552 way ANOVA (Syp: $P<0.001, \mathrm{~F}_{26}=25.05$; PSD95: $P<0.001, \mathrm{~F}_{26}=19.92$ ) and Bonferroni's

553 multiple-comparisons test. ns, not significant; ${ }^{* \star} P<0.001$.

554

555

556

557

558

559

560

561

562

563

564

565

566

567

568

569

570 
Fig. 5: Selective neural IFNAR ablation reduces plaque load and prevents pre-synaptic loss.

572 a, Representative images of Stat1 expression in plaque environment in 5XFAD animals with

573 neural-specific deletion of Ifnar1 (“5XFAD;NKO”; $n=7$ animals) compared to Ifnar1-sufficient

574 5XFAD animals (“5XFAD”; $n=7$ animals) (insets show isolated Stat 1 channel in outlined $\mathrm{NeuN}^{+}$

575 neuronal nuclei; scale bar, $30 \mu \mathrm{m})$.

b, Histological examination of plaque burden in plaque-bearing regions of $5 \operatorname{XFAD}(n=9$ animals), 5XFAD;MKO ( $n=9$ animals), and 5XFAD;NKO ( $n=7$ animals) using thioflavin $S$ to mark dense core plaques, and 6E10 antibody to mark all A $\beta$ fibrils (scale bar, $500 \mu \mathrm{m}$ ). Quantification (below) of percent area of plaque markers by brain region. Data represent means and s.e.m. Statistics were performed with two-tailed $t$-tests. ns, not significant; ${ }^{*} P<0.05 ;{ }^{* *} P<0.01$.

c, Representative images and quantification of Ifitm3 signals localized inside LAMP1+ DNs and $\mathrm{GFAP}^{+}$astrocytes (scale bar, $20 \mu \mathrm{m}$ ). 5XFAD, $n=4$ animals; 5XFAD;MKO, $n=4$ animals; 5XFAD;NKO, $n=5$ animals. Data represent means and s.e.m. Statistics were performed with two-tailed $t$-tests. ns, not significant; ${ }^{* * *} P<0.001$.

d, High-magnification confocal images (scale bar, $3 \mu \mathrm{m}$ ) and relative density quantifications of pre- and post-synaptic puncta marked by synaptophysin and PSD95, respectively, in subicula of Ctrl ( $n=9$ animals), Ctrl;NKO ( $n=3$ animals), 5XFAD ( $n=7$ animals), and 5XFAD;NKO $(n=7$ animals). Data represent means and s.e.m. Statistics were performed with ordinary one-way ANOVA (Syp: $P<0.001, \mathrm{~F}_{22}=14.72$; PSD95: $P<0.001, \mathrm{~F}_{22}=21.26$ ) and Bonferroni's multiplecomparisons test. ns, not significant; ${ }^{* * *} P<0.001$.

e, High-magnification confocal images (scale bar, $3 \mu \mathrm{m}$ ) and quantification of pStat1 ${ }^{+}$puncta colocalized with Syp ${ }^{+}$synapses in subiculum. Ctrl, $n=4$ animals; Ctrl;NKO, $n=3$ animals; 5XFAD, 
bioRxiv preprint doi: https://doi.org/10.1101/2021.11.01.466525. this version posted November 3, 2021. The copyright holder for this preprint (which was not certified by peer review) is the author/funder, who has granted bioRxiv a license to display the preprint in perpetuity. It is made available under aCC-BY-NC-ND 4.0 International license.

594 performed with ordinary one-way ANOVA $\left(P<0.001, \mathrm{~F}_{16}=16.99\right)$ and Bonferroni's multiple-

595 comparisons test. ns, not significant; ${ }^{* \star} P<0.01 ;{ }^{* * *} P<0.001$.

596

597

598

599

600

601

602

603

604

605

606

607

608

609

610

611

612

613 
614 Fig. 6: Memory impairment correlates with IFN signaling, synaptic pathology, and 615 microglial reactivity.

616 a, Nanostring gene expression analysis of hippocampal tissues from IgG- or alFNAR-treated 617 control or 5XFAD animals ( 1 animal per row; Ctrl + IgG, $n=9$ animals; Ctrl + alFNAR, $n=8$ 618 animals; 5 XFAD + IgG, $n=8$ animals; 5XFAD + alFNAR, $n=10$ animals). Top lanes: Genes 619 identified as ISGs are marked with green. All genes are ranked (left to right) by their Pearson 620 correlation $(r)$ with impaired performance in $Y$ maze. Correlations with NOR are also included. 621 Several top inverse correlates are highlighted at bottom.

622 b-e, Correlation analyses (Pearson $r$ ) of $Y$ maze performance with numerous histopathological 623 and transcriptional ( $n=34$ animals profiled) readouts from control and 5XFAD animals treated 624 with IgG or alFNAR ( $n=46$ total animals, groups as listed in a), including synaptic densities (b, $n$ $625=46$ animals), plaque-related parameters (c, $n=22$ animals), neuritic pathology parameters (d, $626 n=22$ animals), and microglial activation markers (e, $n=46$ animals). Simple linear regression

627 lines (solid) with 95\% Cl intervals (dashed) were added to plots, as are Pearson $r$ and associated $628 P$ values. Plots were colored by $r$ value as follows: $1>r>0.65$, yellow; $0.65>r>0.55$, light green; $6290.55>r>0.45$, dark green; $0.45>r>0.35$, blue; $r<0.35$, dark purple. 
631

\section{Fig. 7: Post-synapses are preferential engulfed by IFN-stimulated $\mathrm{Axl}^{+}$microglia}

a, Representative images of microglia in relation to synaptic markers in subicula of treated 5XFAD animals as in Figure 2 (scale bar, $5 \mu \mathrm{m}$ ). Quantification of microglial engulfment of both markers. Ctrl + IgG, $n=13$ animals; Ctrl + alFNAR, $n=11$ animals; 5XFAD + IgG, $n=11$ animals; 5XFAD + alFNAR, $n=11$ animals. Data represent means and s.e.m. Statistics were performed with ordinary one-way ANOVA (Syp: $P=0.0717, F_{42}=2.510$; PSD95: $P<0.001, F_{42}=19.23$ ) and Bonferroni's multiple-comparisons test. ns, not significant; ${ }^{* \star \star} P<0.001$.

b, Quantification of microglial engulfment of pre- and post-synaptic puncta in subicula of $\operatorname{Ctrl}$ ( $n=$ 5 animals for Syp, $n=9$ animals for PSD95), Ctrl;MKO ( $n=2$ animals for Syp, $n=6$ animals for PSD95), 5XFAD ( $n=3$ animals for Syp, $n=7$ animals for PSD95), and 5XFAD;MKO ( $n=4$ animals for Syp, $n=8$ animals for PSD95). Data represent means and s.e.m. Statistics were performed with ordinary one-way ANOVA (Syp: $P=0.688, F_{10}=0.5038$; PSD95: $P<0.001, F_{26}=$ 44.28) and Bonferroni's multiple-comparisons test. ns, not significant; ${ }^{* \star *} P<0.001$.

c, Quantification of relative uptake of PSD95 ${ }^{+}$post-synaptic puncta by GFP- $(n=19$ cells) and $\mathrm{GFP}^{+}$microglia ( $n=21$ cells) from 5-month-old 5XFAD;MxG animals ( $n=3$ animals). Data are presented as a violin plot with medians (dashed lines) and quartiles (dotted lines). Statistics were performed with two-tailed $t$-test. ${ }^{* *} P<0.001$.

d, Representative high-magnification confocal images of $\mathrm{PSD95}^{+}$post-synapses in proximity ( $\leq 200 \mathrm{~nm}$ ) to TAM receptors Axl and Mer, and TAM ligand Gas6 (scale bar, $2 \mu \mathrm{m}$ ). Quantification of co-localization, as relative percent of PSD95 puncta, of the three molecules with PSD95 (AxI + PSD95: $n=13$ Ctrl images, $n=17$ 5XFAD images; Mer + PSD95: $n=14$ Ctrl images, $n=15$ 5XFAD images; Gas6 + PSD95: $n=7$ Ctrl images, $n=6$ 5XFAD images). Data represent means and s.e.m. of images from $n=4$ animals per genotype. Statistics were performed with two-tailed $t$-tests. ${ }^{* \star *} P<0.001$. 
e, Representative high-magnification confocal image of $\mathrm{Axl}^{+}$microglial processes contacting dendritic spines (arrow) in a 5XFAD mouse on the Thy1-eGFP background (scale bar, $2 \mu \mathrm{m}$ ), and quantification of relative frequency of observed contacts between control $(n=42$ dendrites $>10$ $\mu \mathrm{m}$ long from $n=8$ animals) and 5XFAD mice ( $n=44$ dendrites $>10 \mu \mathrm{m}$ long from $n=8$ animals). Data represent means and s.e.m. Statistics were performed with two-tailed $t$-test. ${ }^{* *} P<0.001$.

f, Representative confocal image of $\mathrm{Clec7a}^{+}$microglia with varying degrees of $\mathrm{Axl}$ expression in relation to PSD95 ${ }^{+}$post-synapses in a 5-month-old 5XFAD animal (scale bar, $20 \mu \mathrm{m}$ ). Histological analysis of single microglia in both control and 5XFAD brains stratified by levels of Clec7a and Axl expression, showing relative amounts of $\mathrm{PSD}^{+} 5^{+}$synaptic uptake in each category. Ctrl

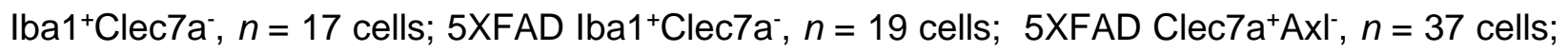

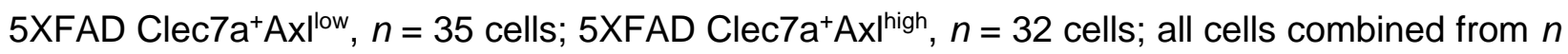
$=2$ Ctrl animals and $n=3$ XXFAD animals at 5 months. Data are presented as a violin plot with medians (dashed lines) and quartiles (dotted lines). Statistics were performed with ordinary oneway ANOVA $\left(P<0.001, \mathrm{~F}_{135}=95.97\right)$ with Bonferroni's multiple-comparisons test. ns, not significant; ${ }^{* *} P<0.001$. Pearson $r$ at right was calculated by correlation analysis of $A x I$ expression and PSD95 ${ }^{+}$uptake in all Clec7a ${ }^{+}$cells.

g, Quantified co-localization of Axl and Gas6 with PSD95, as relative percent of the synaptic 672 puncta, in 5XFAD animals treated with $\operatorname{lgG}(n=11$ animals) or aIFNAR $(n=11$ animals), and in 673 Ifnar1 microglia conditional KO lines (5XFAD, $n=7$ animals; 5XFAD;MKO, $n=8$ animals). Data 674 represent means and s.e.m. Statistics were performed with two-tailed $t$-tests. ${ }^{\star *} P<0.01$; 675 ${ }^{* \star *} P<0.001$.

h, Correlation analyses (Pearson $r$ ) of post-synaptic density, engulfment, and $\mathrm{Y}$ maze 677 performance with the extent of Axl expression in microglia by histological analysis in 5XFAD 678 animals treated with IgG or alFNAR ( $n=46$ total animals, groups as listed in Figure 6a). Simple 
bioRxiv preprint doi: https://doi.org/10.1101/2021.11.01.466525 this version posted November 3,2021. The copyright holder for this preprint (which was not certified by peer review) is the author/funder, who has granted bioRxiv a license to display the preprint in perpetuity. It is made available under aCC-BY-NC-ND 4.0 International license.

679 linear regression lines (solid) with $95 \% \mathrm{Cl}$ intervals (dashed) were added to plots, as are Pearson

$680 \quad r$ and associated $P$ values.

681

682

683

684

685

686

687

688

689

690

691

692

693 


\section{References}

695 1. B. De Strooper, E. Karran, The Cellular Phase of Alzheimer's Disease. Cell 164, 603-615 (2016).

696 2. B. Zhang et al., Integrated systems approach identifies genetic nodes and networks in late-onset

697 Alzheimer's disease. Cell 153, 707-720 (2013).

698 3. K. L. Huang et al., A common haplotype lowers PU.1 expression in myeloid cells and delays onset of Alzheimer's disease. Nat. Neurosci. 20, 1052-1061 (2017).

700 4. E. R. Roy et al., Type I interferon response drives neuroinflammation and synapse loss in

701

702 Alzheimer disease. J. Clin. Invest. 130, 1912-1930 (2020).

703

704

705

5. J. Y. Hur et al., The innate immunity protein IFITM3 modulates gamma-secretase in Alzheimer's disease. Nature 586, 735-740 (2020).

6. C. Sala Frigerio et al., The Major Risk Factors for Alzheimer's Disease: Age, Sex, and Genes Modulate the Microglia Response to Abeta Plaques. Cell Rep 27, 1293-1306 e1296 (2019).

7. M. Olah et al., Single cell RNA sequencing of human microglia uncovers a subset associated with Alzheimer's disease. Nat Commun 11, 6129 (2020).

8. D. A. Salih et al., Genetic variability in response to amyloid beta deposition influences Alzheimer's disease risk. Brain Commun 1, fcz022 (2019).

9. O. Korvatska et al., Triggering Receptor Expressed on Myeloid Cell 2 R47H Exacerbates Immune Response in Alzheimer's Disease Brain. Frontiers in immunology 11, 559342 (2020).

10. J. Di Domizio et al., Nucleic acid-containing amyloid fibrils potently induce type I interferon and stimulate systemic autoimmunity. Proc Natl Acad Sci U S A 109, 14550-14555 (2012).

11. N. E. Propson, E. R. Roy, A. Litvinchuk, J. Kohl, H. Zheng, Endothelial C3a receptor mediates vascular inflammation and blood-brain barrier permeability during aging. J. Clin. Invest. 131 (2021).

12. H. Keren-Shaul et al., A Unique Microglia Type Associated with Restricting Development of Alzheimer's Disease. Cell 169, 1276-1290 e1217 (2017).

13. S. Krasemann et al., The TREM2-APOE Pathway Drives the Transcriptional Phenotype of Dysfunctional Microglia in Neurodegenerative Diseases. Immunity 47, 566-581 e569 (2017).

14. P. Ejlerskov et al., Lack of Neuronal IFN-beta-IFNAR Causes Lewy Body- and Parkinson's Diseaselike Dementia. Cell 163, 324-339 (2015).

15. M. R. Minter et al., Deletion of the type-1 interferon receptor in APPSWE/PS1DeltaE9 mice preserves cognitive function and alters glial phenotype. Acta neuropathologica communications 4, 72 (2016).

16. M. Yasuda, S. Nagappan-Chettiar, E. M. Johnson-Venkatesh, H. Umemori, An activity-dependent determinant of synapse elimination in the mammalian brain. Neuron 109, 1333-1349 e1336 (2021).

17. J. D. Crapser et al., Microglia facilitate loss of perineuronal nets in the Alzheimer's disease brain. EBioMedicine 58, 102919 (2020).

18. G. Lemke, How macrophages deal with death. Nat Rev Immunol 19, 539-549 (2019).

19. Y. Huang et al., Microglia use TAM receptors to detect and engulf amyloid beta plaques. Nat. Immunol. 22, 586-594 (2021).

20. C. Fritz-French, W. Tyor, Interferon-alpha (IFNalpha) neurotoxicity. Cytokine Growth Factor Rev. 23, 7-14 (2012).

21. L. S. Zheng et al., Mechanisms for interferon-alpha-induced depression and neural stem cell dysfunction. Stem Cell Reports 3, 73-84 (2014).

22. R. M. Koffie, B. T. Hyman, T. L. Spires-Jones, Alzheimer's disease: synapses gone cold. Molecular neurodegeneration 6, 63 (2011). 
23. M. Prinz, T. Masuda, M. A. Wheeler, F. J. Quintana, Microglia and Central Nervous SystemAssociated Macrophages-From Origin to Disease Modulation. Annu. Rev. Immunol. 39, 251-277 (2021).

24. T. R. Hammond et al., Single-Cell RNA Sequencing of Microglia throughout the Mouse Lifespan and in the Injured Brain Reveals Complex Cell-State Changes. Immunity 50, 253-271 e256 (2019).

25. L. Sebastian Monasor et al., Fibrillar Abeta triggers microglial proteome alterations and dysfunction in Alzheimer mouse models. Elife 9, doi: 10.7554/eLife.54083 (2020).

26. W. T. Chen et al., Spatial Transcriptomics and In Situ Sequencing to Study Alzheimer's Disease. Cell 182, 976-991 e919 (2020).

27. W. Li et al., Microglia have a more extensive and divergent response to interferon-alpha compared with astrocytes. Glia 66, 2058-2078 (2018).

28. H. Cho et al., Differential innate immune response programs in neuronal subtypes determine susceptibility to infection in the brain by positive-stranded RNA viruses. Nat. Med. 19, 458-464 (2013).

29. S. A. Liddelow et al., Neurotoxic reactive astrocytes are induced by activated microglia. Nature 541, 481-487 (2017).

30. J. B. Altman et al., Type I IFN is siloed in endosomes. Proc Natl Acad Sci U S A 117, 17510-17512 (2020).

31. K. R. Sadleir et al., Presynaptic dystrophic neurites surrounding amyloid plaques are sites of microtubule disruption, BACE1 elevation, and increased Abeta generation in Alzheimer's disease. Acta Neuropathol 132, 235-256 (2016).

32. R. Sanchez-Varo et al., Abnormal accumulation of autophagic vesicles correlates with axonal and synaptic pathology in young Alzheimer's mice hippocampus. Acta Neuropathol 123, 53-70 (2012).

33. H. J. Cho et al., IFN-gamma-induced BACE1 expression is mediated by activation of JAK2 and ERK1/2 signaling pathways and direct binding of STAT1 to BACE1 promoter in astrocytes. Glia 55, 253-262 (2007).

34. H. J. Cho et al., Constitutive JAK2/STAT1 activation regulates endogenous BACE1 expression in neurons. Biochem. Biophys. Res. Commun. 386, 175-180 (2009).

35. W. L. Hsu, Y. L. Ma, D. Y. Hsieh, Y. C. Liu, E. H. Lee, STAT1 negatively regulates spatial memory formation and mediates the memory-impairing effect of Abeta. Neuropsychopharmacology: official publication of the American College of Neuropsychopharmacology 39, 746-758 (2014).

36. D. J. Tai, W. L. Hsu, Y. C. Liu, Y. L. Ma, E. H. Lee, Novel role and mechanism of protein inhibitor of activated STAT1 in spatial learning. EMBO J. 30, 205-220 (2011).

37. D. K. Wilton, L. Dissing-Olesen, B. Stevens, Neuron-Glia Signaling in Synapse Elimination. Annu. Rev. Neurosci. 42, 107-127 (2019).

38. Q. Shi et al., Complement C3 deficiency protects against neurodegeneration in aged plaque-rich APP/PS1 mice. Sci Transl Med 9, eaaf6295 (2017).

39. T. Wu et al., Complement C3 Is Activated in Human AD Brain and Is Required for Neurodegeneration in Mouse Models of Amyloidosis and Tauopathy. Cell Rep 28, 2111-2123 e2116 (2019).

40. S. Hong et al., Complement and microglia mediate early synapse loss in Alzheimer mouse models. Science 352, 712-716 (2016).

41. F. Filipello et al., The Microglial Innate Immune Receptor TREM2 Is Required for Synapse Elimination and Normal Brain Connectivity. Immunity 48, 979-991 e978 (2018).

42. L. Cheadle et al., Sensory Experience Engages Microglia to Shape Neural Connectivity through a Non-Phagocytic Mechanism. Neuron 108, 451-468 e459 (2020). 
43. T. Li et al., A splicing isoform of GPR56 mediates microglial synaptic refinement via phosphatidylserine binding. EMBO J. 39, e104136 (2020).

44. N. Scott-Hewitt et al., Local externalization of phosphatidylserine mediates developmental synaptic pruning by microglia. EMBO J. 39, e105380 (2020). cues microglia to feast: Two new studies identify how a common apoptotic cell flag is used to sculpt neural circuits. EMBO J. 39, e105924 (2020).

800

801

802

46. W.-S. Chung et al., Astrocytes mediate synapse elimination through MEGF10 and MERTK pathways. Nature 504, 394 (2013).

47. P. P. Sainaghi et al., Growth Arrest Specific 6 Concentration is Increased in the Cerebrospinal Fluid of Patients with Alzheimer's Disease. Journal of Alzheimer's disease : JAD 55, 59-65 (2017).

48. N. Mattsson et al., CSF protein biomarkers predicting longitudinal reduction of CSF betaamyloid42 in cognitively healthy elders. Transl Psychiatry 3, e293 (2013).

49. Z. He et al., Amyloid-beta plaques enhance Alzheimer's brain tau-seeded pathologies by

803 facilitating neuritic plaque tau aggregation. Nat. Med. 24, 29-38 (2018).

50. S. H. Lee et al., Trem2 restrains the enhancement of tau accumulation and neurodegeneration by beta-amyloid pathology. Neuron 109, 1283-1301 e1286 (2021). 
bioRxiv preprint doi: https://doi.org/10.1101/2021.11.01.466525; this version posted November 3, 2021. The copyright holder for this preprint (which was not certified by peer review) is the author/funder, who has granted bioRxiv a license to display the preprint in perpetuity. It is made available under aCC-BY-NC-ND 4.0 International license.

\section{Fig. 1}
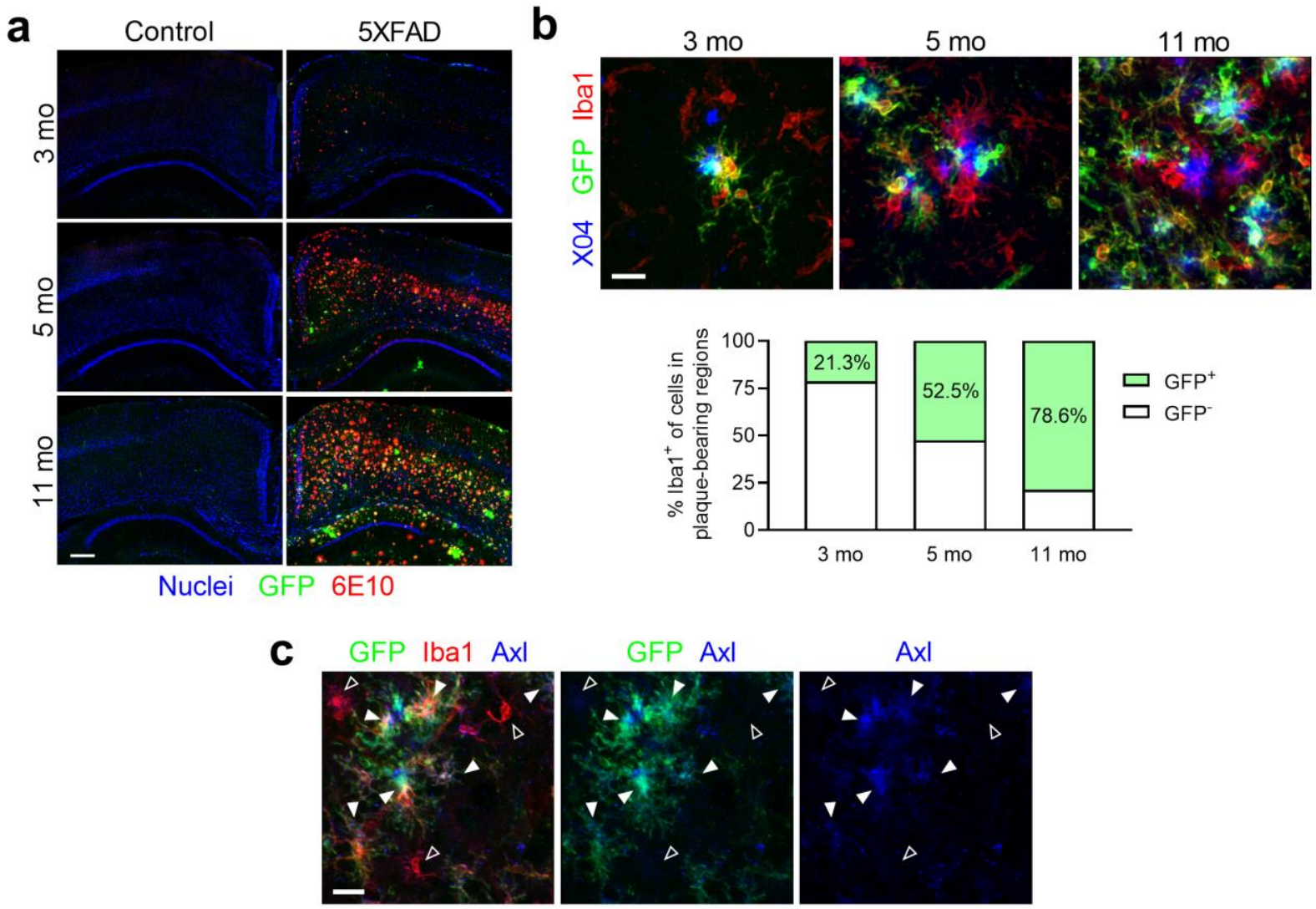

d
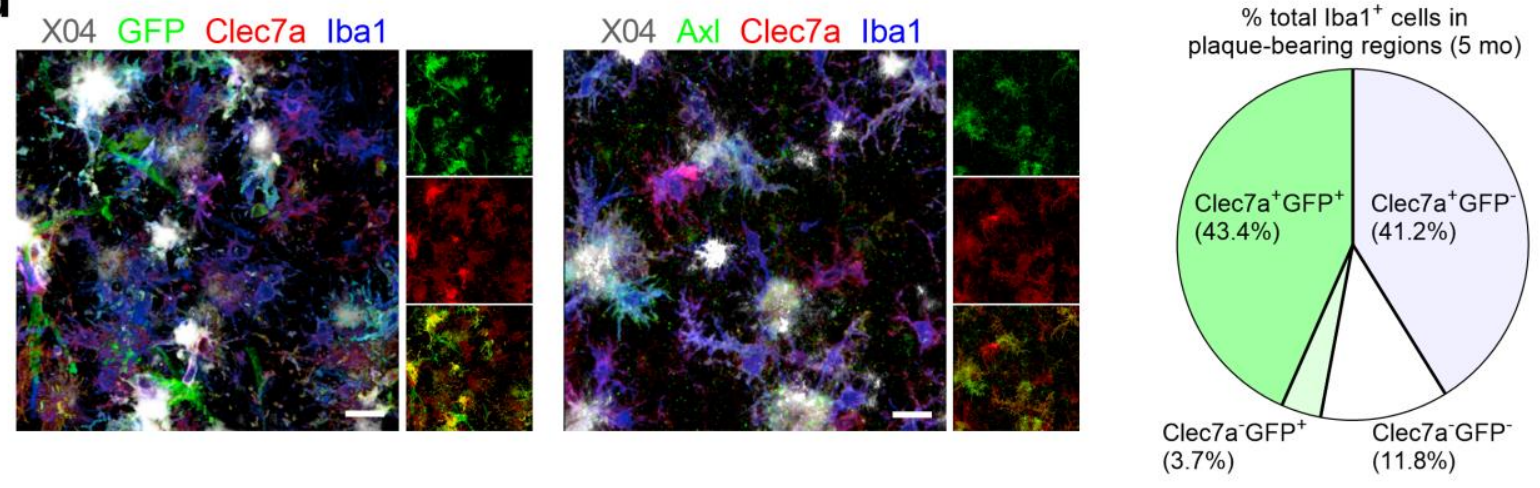

814 
Fig. 2

a

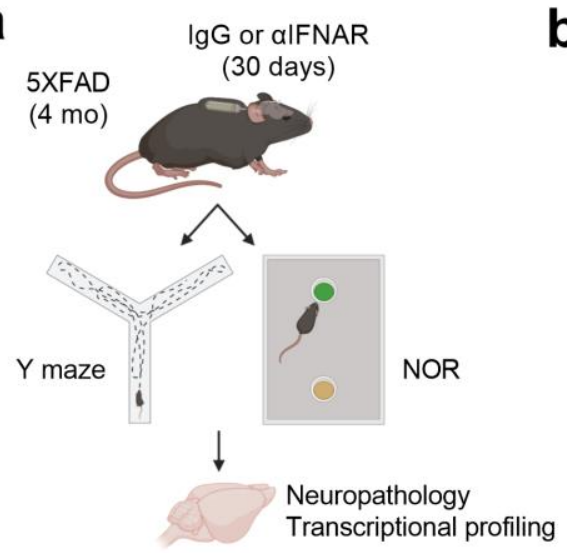

C

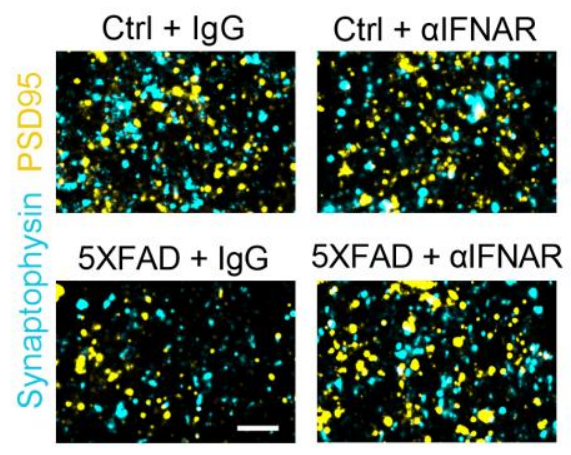

d

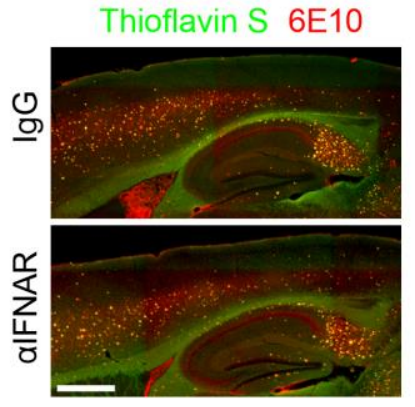

b

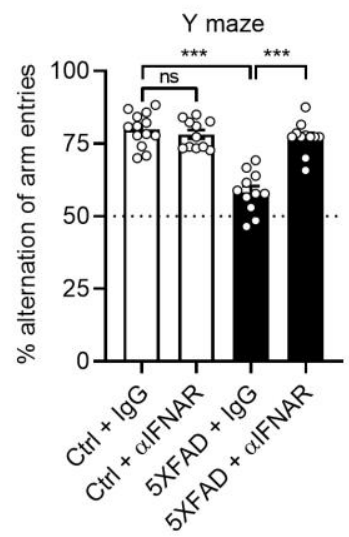

Synaptic puncta densities

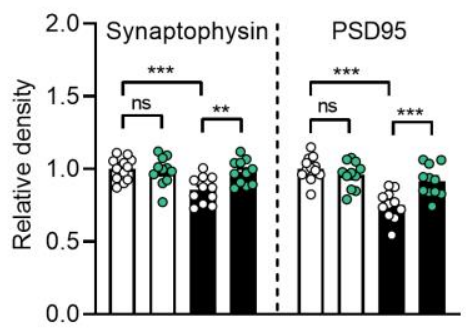

a] $\mathrm{Ctrl}+\lg G$

- $5 X F A D+\lg G$

Q Ctrl + aIFNAR

$6 \mathrm{E} 10$

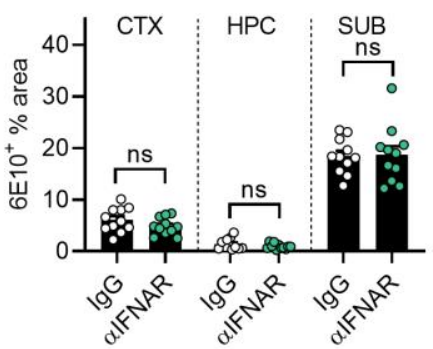

Novel object recognition (NOR)
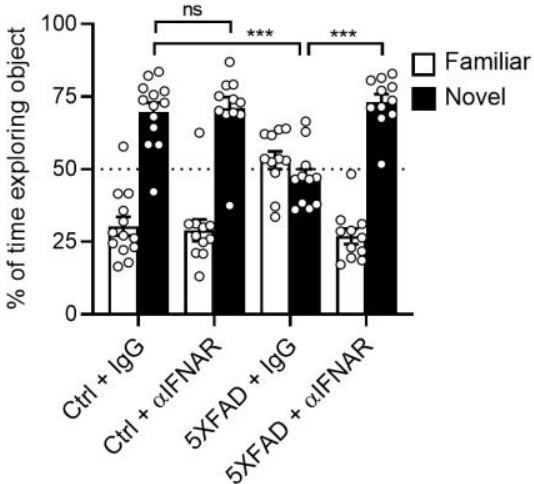
Fig. 3

a

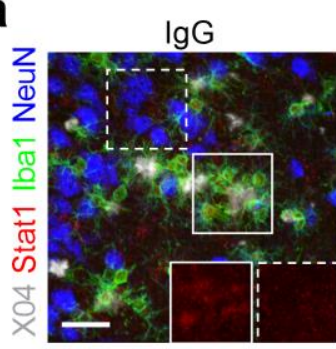

C
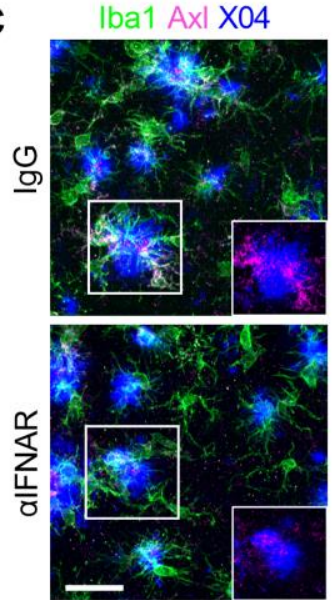

alFNAR

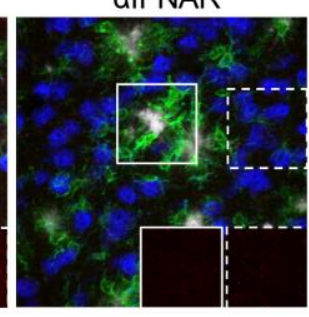

Iba1 Clec7a X04
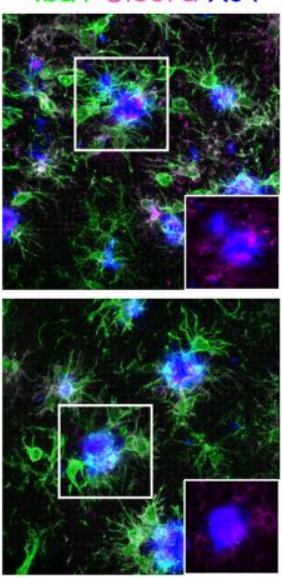

b

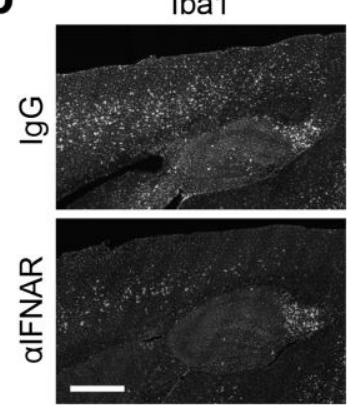

口 $\mathrm{Ctrl}+\lg G$

Q Ctrl + alFNAR

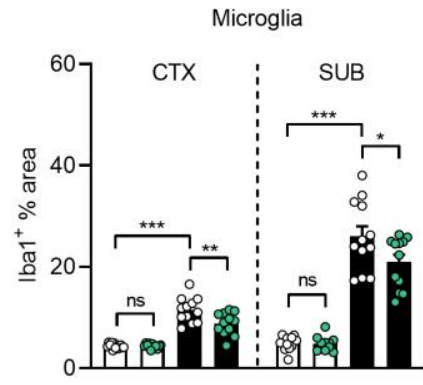

a $5 X F A D+\lg G$

5XFAD + alFNAR

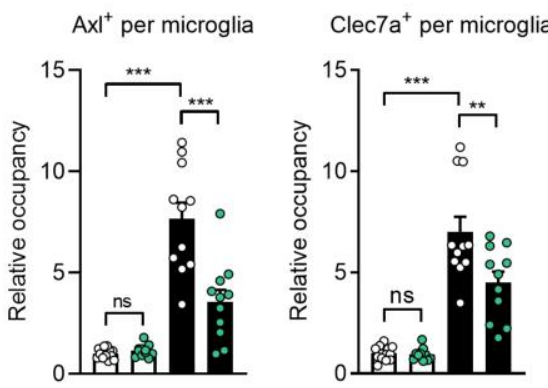

d
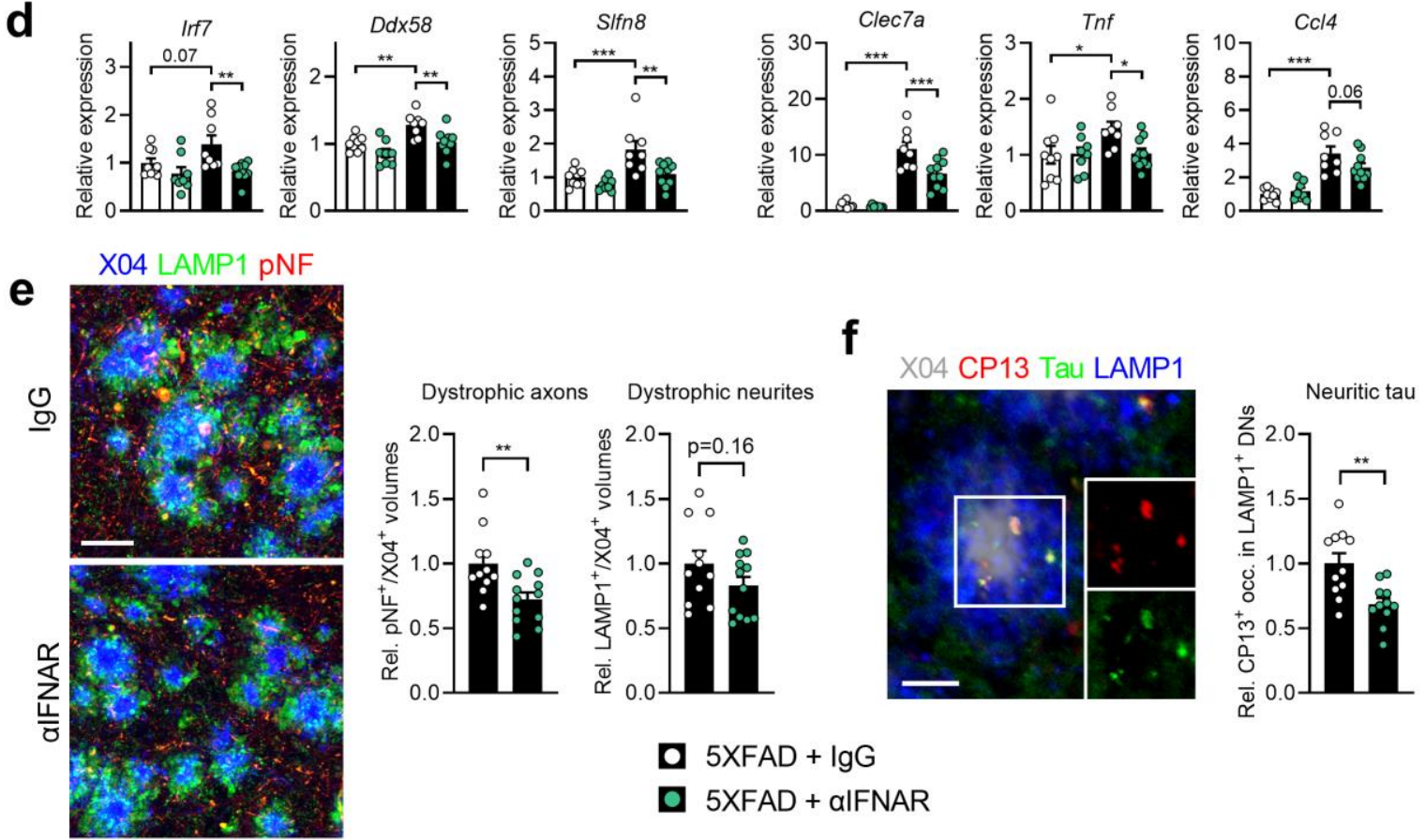
Fig. 4

a
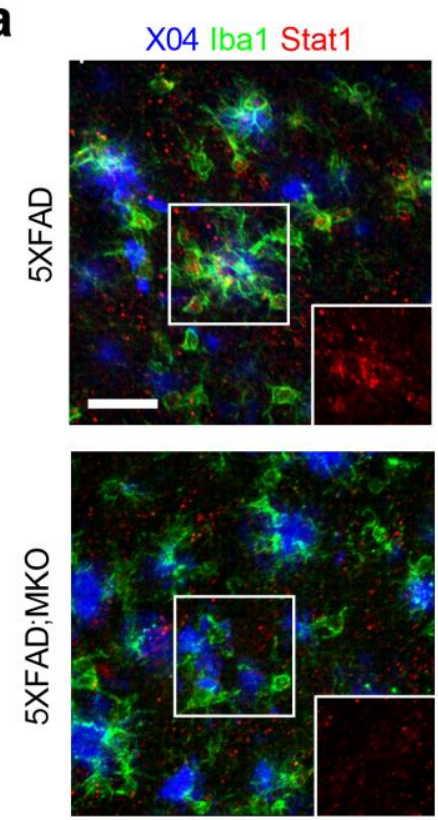

b

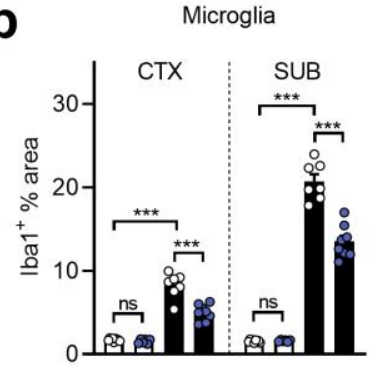

$\mathrm{AxI}^{+}$per microglia

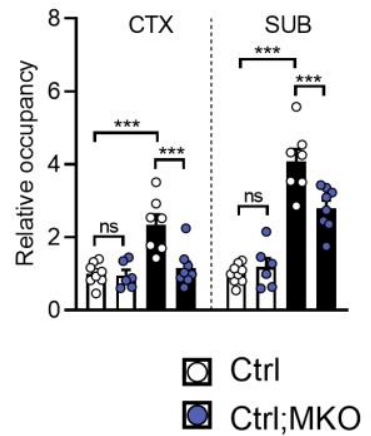

$\mathrm{CD}^{+} 8^{+}$per microglia

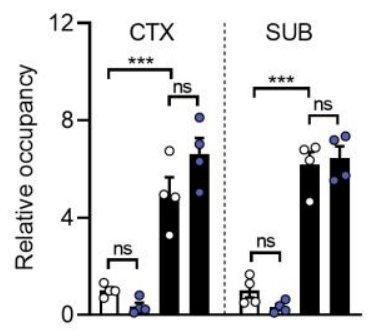

Clec $7 \mathrm{a}^{+}$per microglia

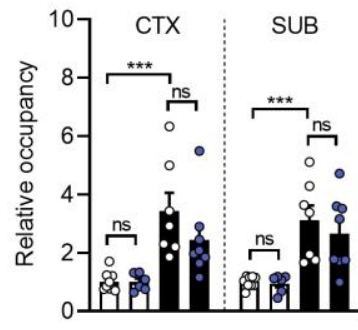

व 5XFAD

DXFAD;MKO
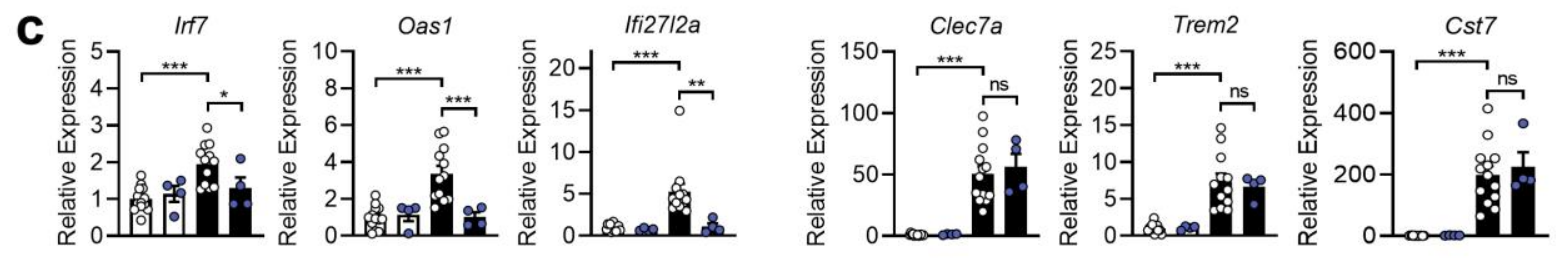

d

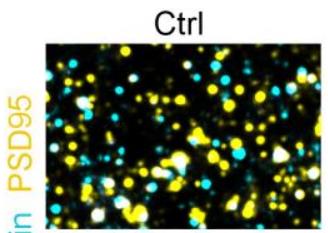

5XFAD

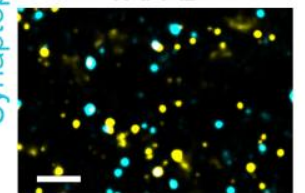

Ctrl;MKO

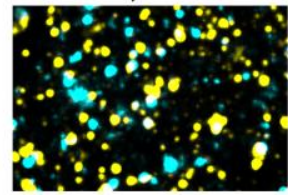

5XFAD;MKO

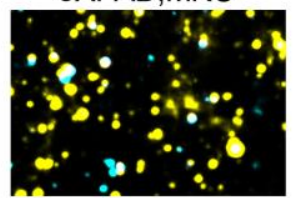

Synaptic puncta densities

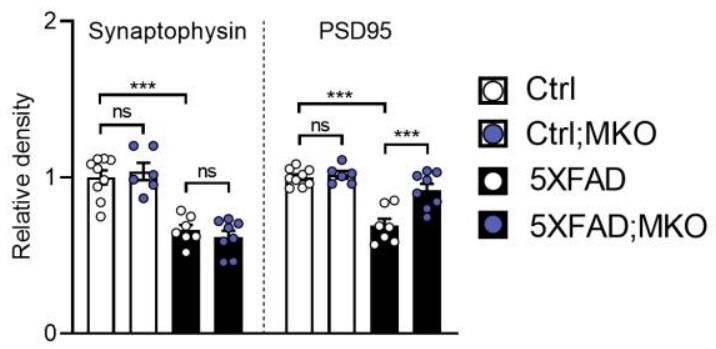


Fig. 5

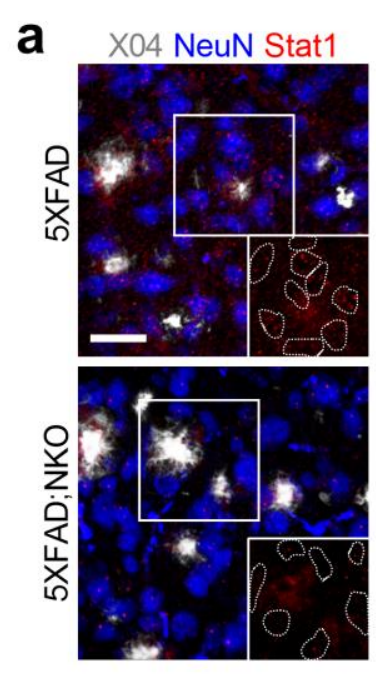

b

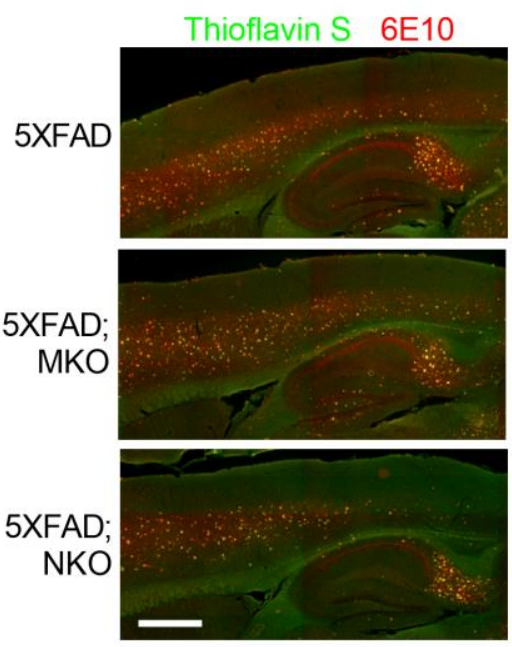

Thioflavin S

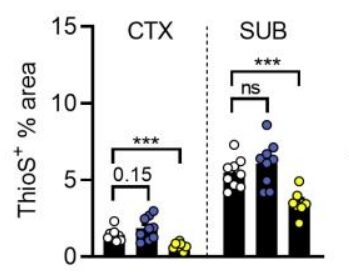

$6 \mathrm{E} 10$
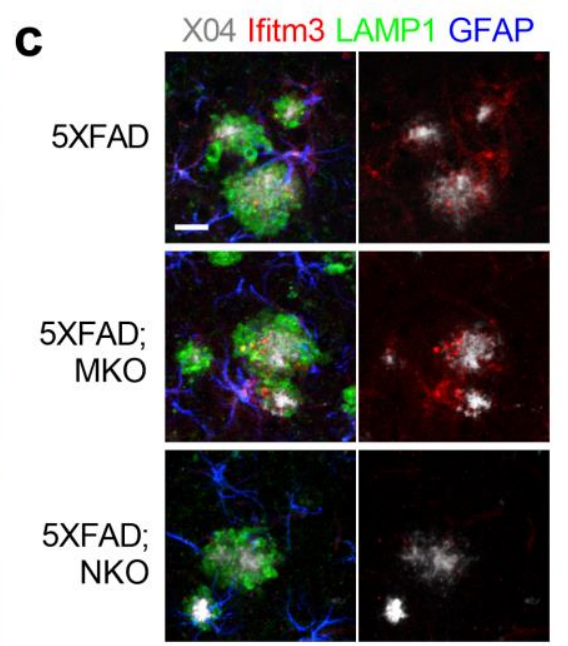

D 5XFAD

5XFAD;MKO

5XFAD;NKO

d

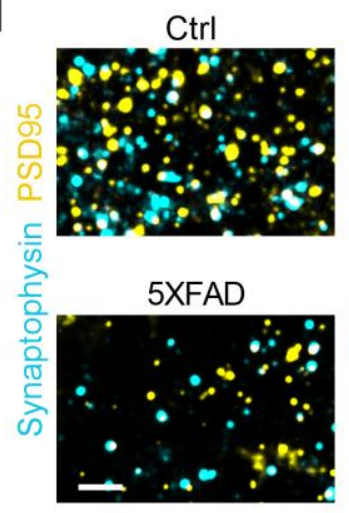

Synaptic puncta densities

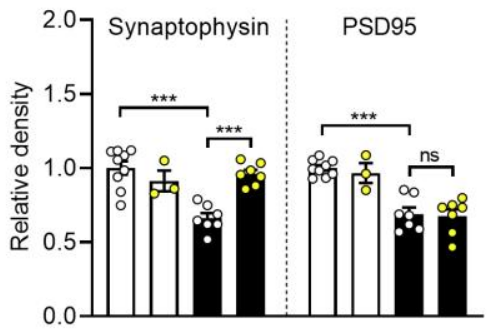

Ctrl;NKO

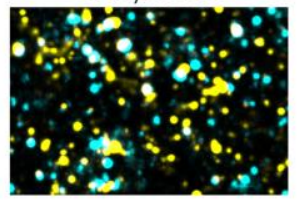

5XFAD;NKO

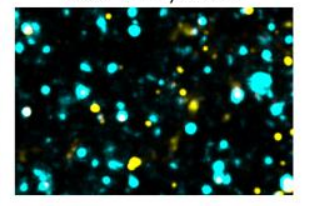

○ Ctrl

Ctrl;NKO

- 5XFAD

D 5XFAD;NKO e

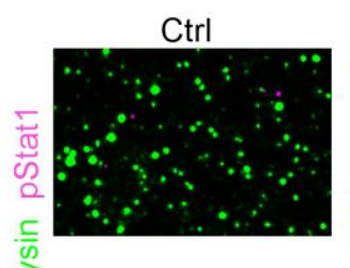

5XFAD

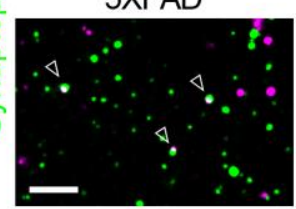

Ifitm $^{+}$signal occupancy

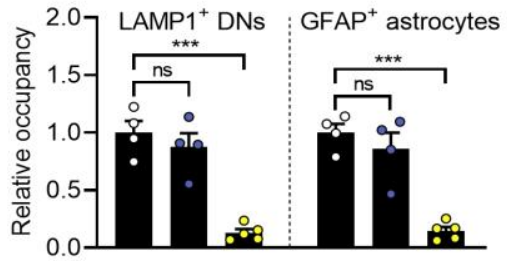

Pre-synaptic pStat1

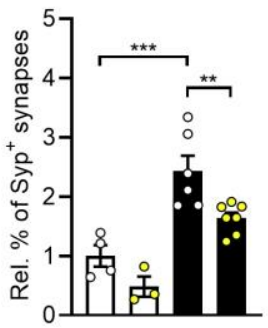


bioRxiv preprint doi: https://doi.org/10.1101/2021.11.01.466525; this version posted November 3, 2021. The copyright holder for this preprint (which was not certified by peer review) is the author/funder, who has granted bioRxiv a license to display the preprint in perpetuity. It is made available under aCC-BY-NC-ND 4.0 International license.

Fig. 6

a

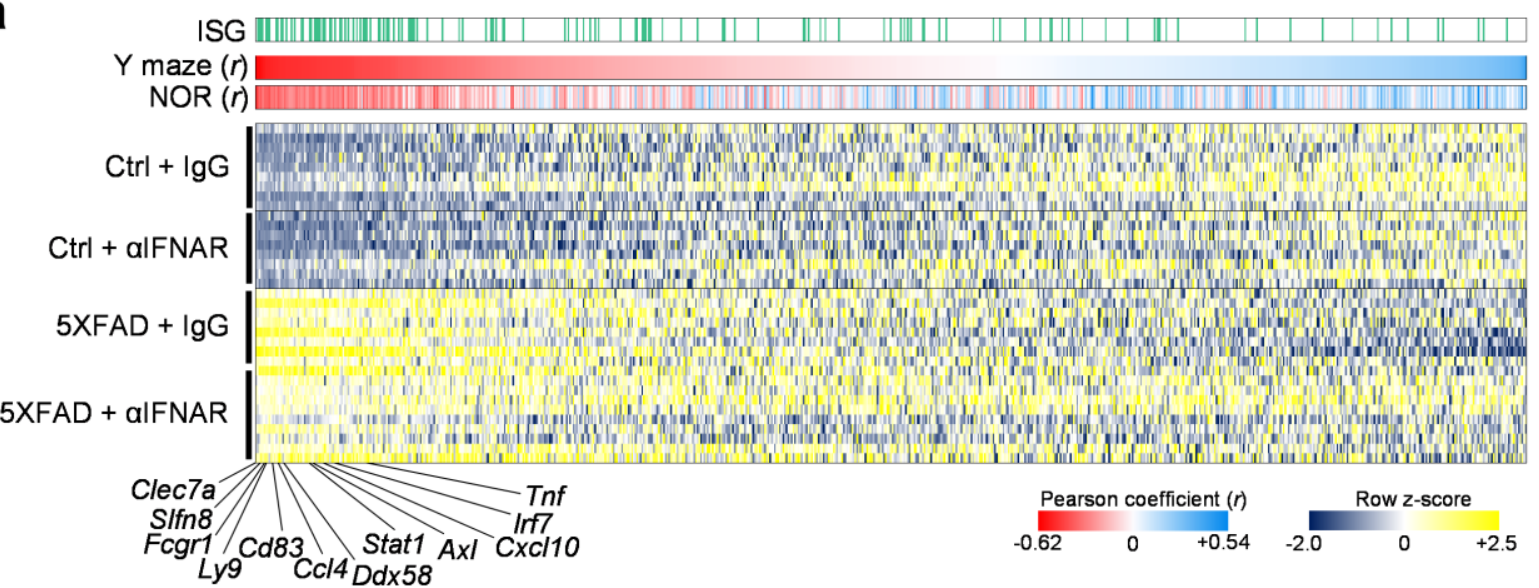

b
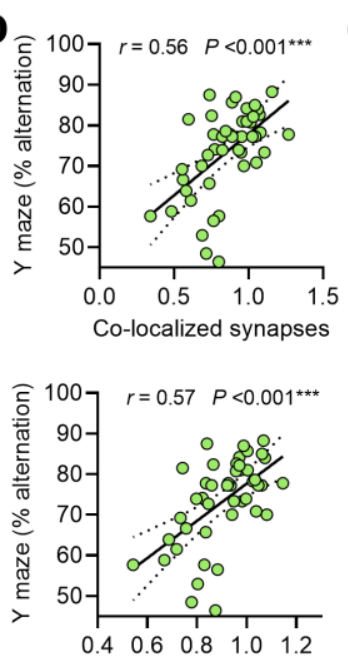

PSD95 ${ }^{+}$synapse density

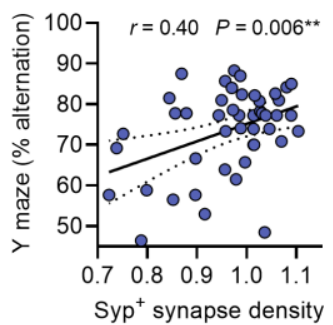

C
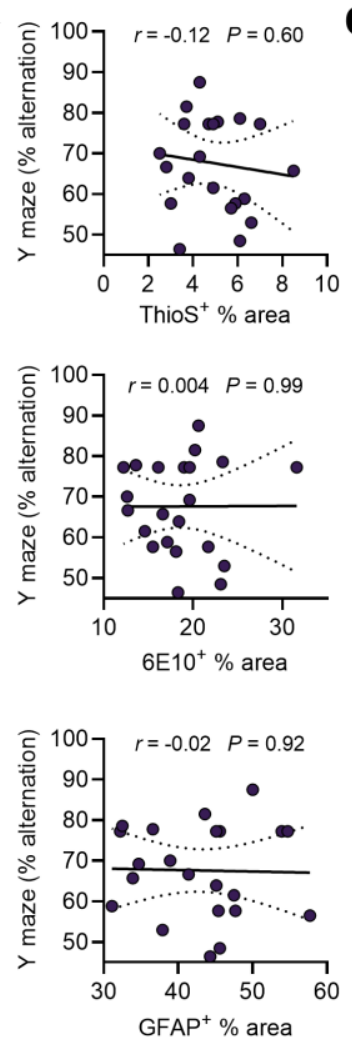

d
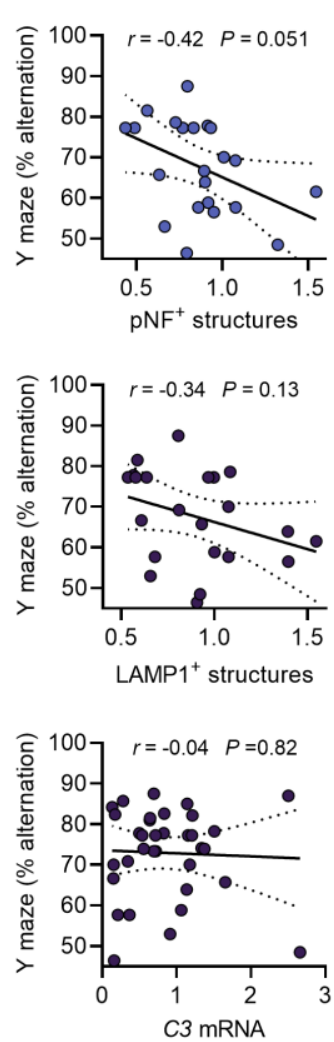

e
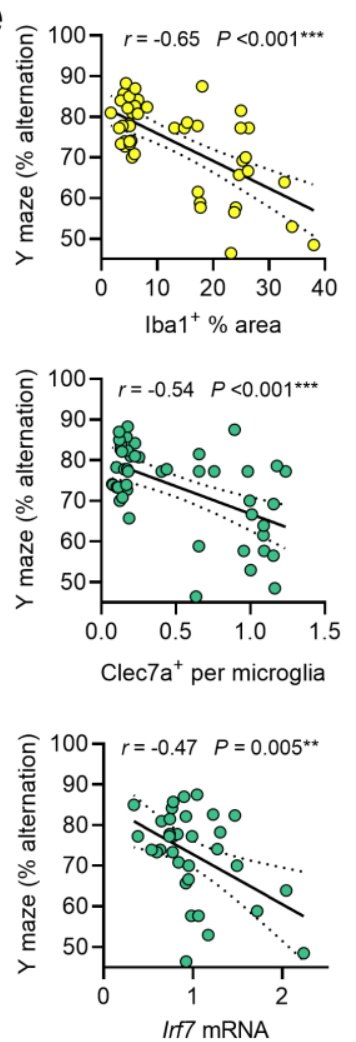


\section{Fig. 7}
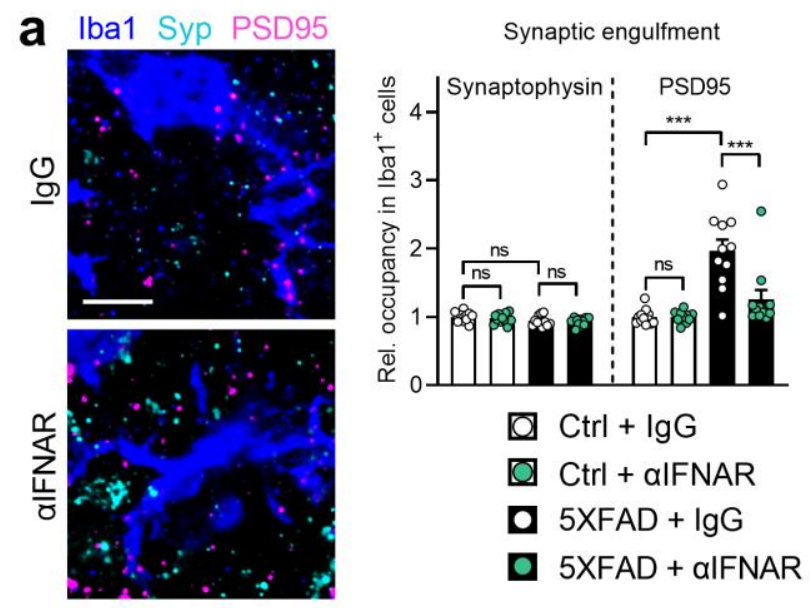

b Synaptic engulfment

C PSD95 engulfment

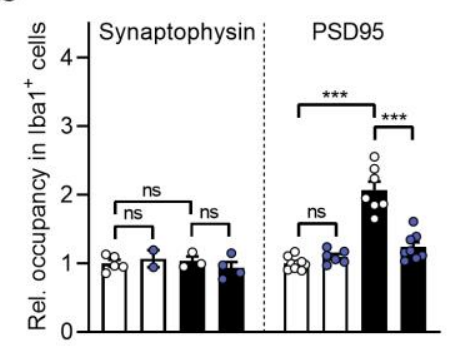

○ Ctrl

回 Ctrl;MKO
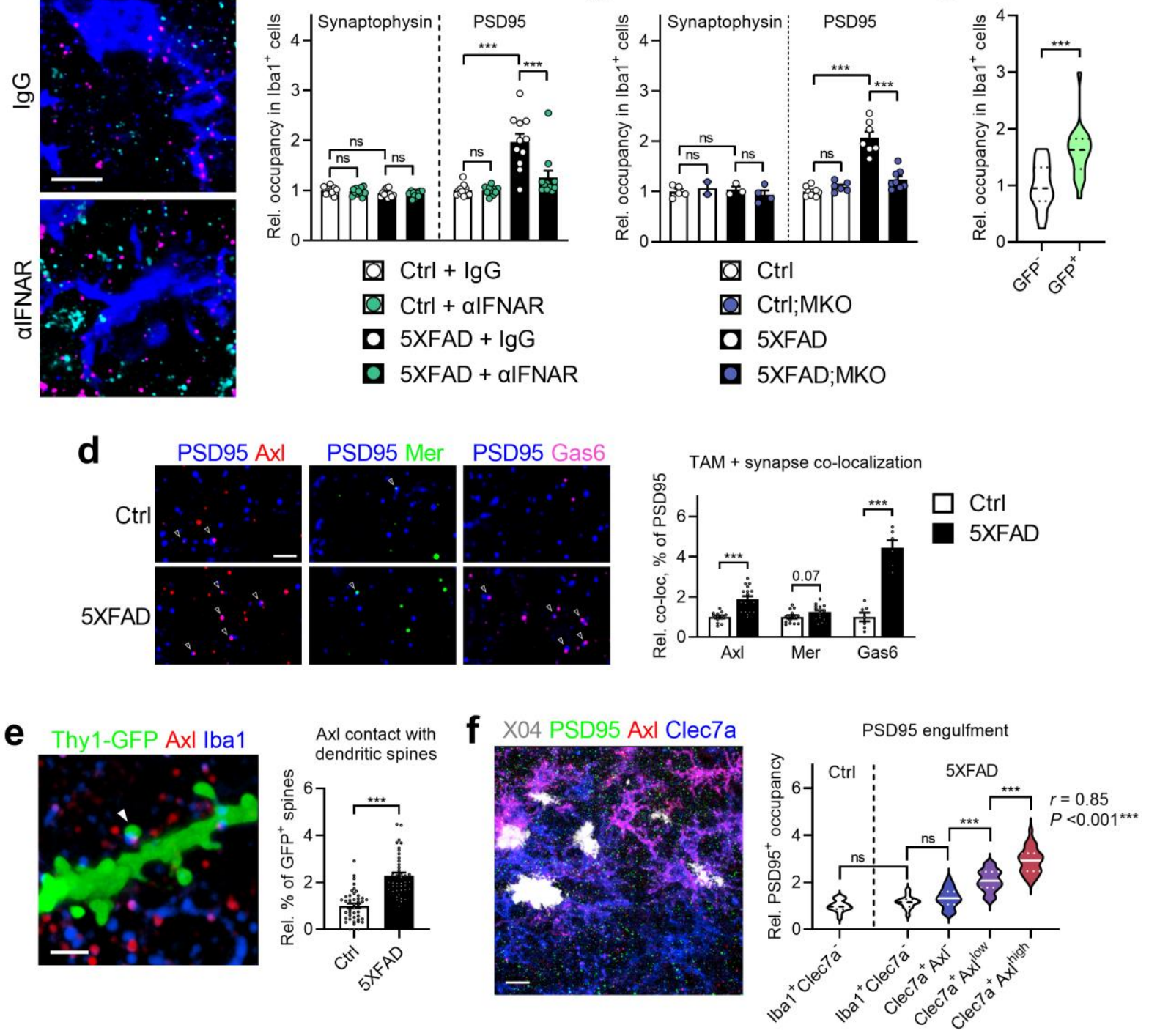

g

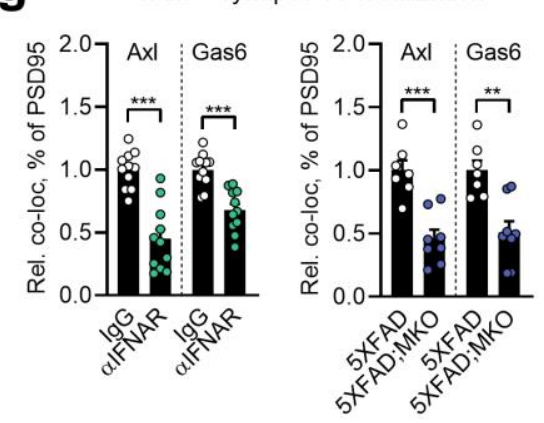

h
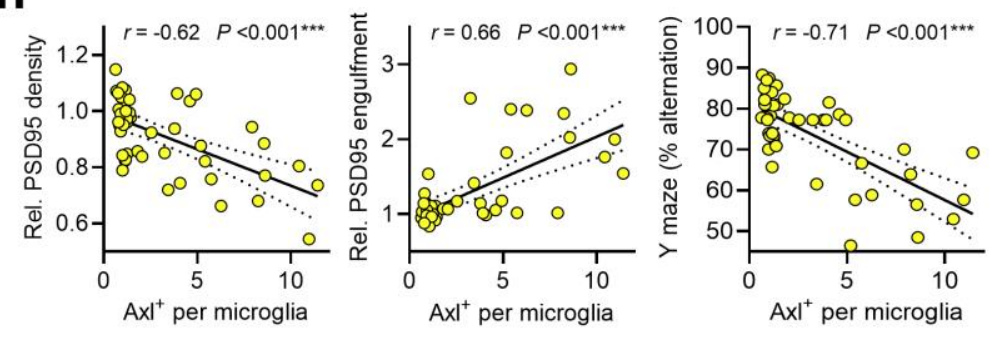